Pure and Applied Mathematics Quarterly

Volume 4, Number 2

(Special Issue: In honor of

Fedor Bogomolov, Part 1 of 2)

427-468, 2008

\title{
Dual Double EPW-sextics and Their Periods
}

\author{
Kieran G. O'Grady \\ Dedicato a Fedya Bogomolov in occasione del $60^{\circ}$ compleanno
}

\section{INTRODUCTION}

Eisenbud, Popescu and Walter have constructed certain singular sextic hypersurfaces (EPW-sextics) in $\mathbb{P}^{5}$ (Example (9.3) of [4]) which come provided with a natural double cover: we have shown [13] that the generic such double cover is a deformation of the Hilbert square of a $K 3$ and that the family of double EPWsextics is a locally versal family of projective deformations of $(K 3)^{[2]}$. Thus the family of double EPW-sextics is similar to the family of Fano varieties of lines on a cubic 4-fold (see [2]), with the following difference: the Plücker ample divisor on the Fano variety of lines has square 6 for the Beauville-Bogomolov quadratic form (see $[1,2]$ ) while the natural polarization of a double EPW-sextic has square 2 (see [13]). Let $Y \subset \mathbb{P}^{5}$ be a generic EPW-sextic: we proved in [13] that the dual $Y^{\vee} \subset\left(\mathbb{P}^{5}\right)^{\vee}$ is another generic EPW-sextic. Thus we may associate to the natural double cover $X$ of $Y$ a "dual" variety $X^{\vee}$ namely the natural double cover of $Y^{\vee}$. This construction defines a (rational) involution on the moduli space of double EPW-sextics. In [13] we showed that a generic EPW-sextic is not self-dual and hence the involution on the moduli space of double EPW-sextics is not the identity; in this paper we determine the relation between the periods of a double EPW-sextic and its dual. Before stating the result we recall the definition of EPW-sextics. Let $V$ be a 6 -dimensional $\mathbb{C}$-vector space. Choose an isomorphism

Received April 30, 2006. 
vol: $\wedge^{6} V \stackrel{\sim}{\longrightarrow} \mathbb{C}$ and let $\omega$ be the symplectic form on $\wedge^{3} V$ defined by wedge product followed by vol. Let $\mathbb{P}(V)$ be the projective space of 1-dimensional sub vector spaces of $V$; then $\omega$ gives $\wedge^{3} V \otimes \mathcal{O}_{\mathbb{P}(V)}$ the structure of a symplectic vectorbundle of rank 20. Let $F$ be the sub-vector-bundle of $\wedge^{3} V \otimes \mathcal{O}_{\mathbb{P}(V)}$ whose fiber $F_{[v]}$ over $[v] \in \mathbb{P}(V)$ consists of tensors divisible by $v$ :

$$
F_{[v]}:=\left\{v \wedge w \mid w \in \wedge^{2} V\right\} .
$$

As is easily checked $F$ is a Lagrangian sub-bundle of $\wedge^{3} V \otimes \mathcal{O}_{\mathbb{P}(V)}$. Let $\mathbb{L} \mathbb{G}\left(\wedge^{3} V\right)$ be the symplectic Grassmannian parametrizing $\omega$-Lagrangian subspaces of $\wedge^{3} V$. For $A \in \mathbb{L} \mathbb{G}\left(\wedge^{3} V\right)$ we let $\lambda_{A}$ be the composition

$$
F \longrightarrow \wedge^{3} V \otimes \mathcal{O}_{\mathbb{P}(V)} \longrightarrow\left(\wedge^{3} V / A\right) \otimes \mathcal{O}_{\mathbb{P}(V)}
$$

and $Y_{A} \subset \mathbb{P}(V)$ be the zero-scheme of $\operatorname{det}\left(\lambda_{A}\right)$; unless $\operatorname{det}\left(\lambda_{A}\right)$ is identically zero ${ }^{1}$ $Y_{A}$ is a sextic hypersurface because $\operatorname{det} F \cong \mathcal{O}_{\mathbb{P}(V)}(-6)$. An EPW-sextic is a hypersurface in $\mathbb{P}(V)$ which is equal to $Y_{A}$ for some $A \in \mathbb{L} \mathbb{G}\left(\wedge^{3} V\right)$. In [13] we described explicitly the non-empty Zariski-open $\mathbb{L} \mathbb{G}\left(\wedge^{3} V\right)^{0} \subset \mathbb{L} \mathbb{G}\left(\wedge^{3} V\right)$ parametrizing $A$ such that $Y_{A}$ has no singularities other than those forced by its description as a degeneracy locus; for $A \in \mathbb{L} \mathbb{G}\left(\wedge^{3} V\right)^{0}$ the singular locus of $Y_{A}$ is a smooth degree-40 irreducible surface and at a singular point $Y_{A}$ is locally (in the classical topology) isomorphic to the product of $\operatorname{sing}\left(Y_{A}\right)$ and an $A_{1}$-singularity. If $A \in \mathbb{L} \mathbb{G}\left(\wedge^{3} V\right)^{0}$ there is a natural double cover $f_{A}: X_{A} \rightarrow Y_{A}$ ramified only over $\operatorname{sing}\left(Y_{A}\right)$, with $X_{A}$ smooth. As shown in [13] the 4-fold $X_{A}$ is a deformation of $(K 3)^{[2]}$ - the Hilbert square of a $K 3$. Let $L_{A}$ be the line-bundle on $X_{A}$ defined by

$$
L_{A}:=f_{A}^{*} \mathcal{O}_{Y_{A}}(1)
$$

then $c_{1}\left(L_{A}\right)$ has square 2 for the Beauville-Bogomolov quadratic form. The family of $\left(X_{A}, L_{A}\right)$ for $A$ varying in $\mathbb{L} \mathbb{G}\left(\wedge^{3} V\right)^{0}$ is a locally complete family of polarized deformations of $(K 3)^{[2]}$. Now we recall the duality map. Let $v^{\vee} l^{\vee}$ be a trivialization of $\wedge^{6} V^{\vee}$ and $\omega^{\vee}$ be the symplectic form on $\wedge^{3} V^{\vee}$ given by wedge-product followed by $v o l^{\vee}$ : let $\mathbb{L} \mathbb{G}\left(\wedge^{3} V^{\vee}\right)$ be the symplectic Grassmannian parametrizing $\omega^{\vee}$-Lagrangian subspaces of $\wedge^{3} V^{\vee}$. For $A \in \mathbb{L} \mathbb{G}\left(\wedge^{3} V\right)$ the

\footnotetext{
${ }^{1}$ If $A$ is the fiber of $F$ over a fixed point then $\operatorname{det}\left(\lambda_{A}\right)$ is identically zero.
} 
annihilator $A^{\perp} \subset \wedge^{3} V^{\vee}$ belongs to $\mathbb{L} \mathbb{G}\left(\wedge^{3} V^{\vee}\right)$. Thus we have an isomorphism

$$
\begin{gathered}
\delta: \mathbb{L} \mathbb{G}\left(\wedge^{3} V\right) \stackrel{\sim}{\longrightarrow} \mathbb{L} \mathbb{G}\left(\wedge^{3} V^{\vee}\right) \\
A \quad \mapsto \quad A^{\perp} .
\end{gathered}
$$

Let

$$
\mathbb{L} \mathbb{G}\left(\wedge^{3} V\right)^{00}:=\mathbb{L} \mathbb{G}\left(\wedge^{3} V\right)^{0} \cap \delta^{-1} \mathbb{L} \mathbb{G}\left(\wedge^{3} V^{\vee}\right)^{0} .
$$

Thus $\mathbb{L} \mathbb{G}\left(\wedge^{3} V\right)^{00}$ is open dense in $\mathbb{L} \mathbb{G}\left(\wedge^{3} V\right)$. Assume that $A \in \mathbb{L} \mathbb{G}\left(\wedge^{3} V\right)^{00}$. By Proposition (3.1) of [13] $Y_{A^{\perp}}$ is $Y_{A}^{\vee}$ (the dual of $Y_{A}$ ); we let

$$
X_{A}^{\vee} \rightarrow Y_{A}^{\vee}=Y_{A^{\perp}}
$$

be the natural double cover and we call $X_{A}^{\vee}$ the dual of $X_{A}$. We will show how to obtain the Hodge structure on $H^{2}\left(X_{A}^{\vee}\right)$ from the Hodge structure on $H^{2}\left(X_{A}\right)$. First we describe the relevant period space. Let $\widetilde{\Lambda}$ be the even lattice

$$
\widetilde{\Lambda}:=U^{3} \widehat{\oplus}\left(-E_{8}\right)^{2} \widehat{\oplus}(-2)
$$

where $\widehat{\oplus}$ denotes orthogonal direct sum, $U$ is the hyperbolic plane and $(-2)$ is the lattice generated by a single element of square -2 ; we will denote by $(\cdot, \cdot)$ the symmetric bilinear form on $\widetilde{\Lambda}$. Let $U$ be one of the hyperbolic planes appearing in Decomposition (1.0.7) and choose

$$
u \in U \text { of square } 2 \text {. }
$$

Let $e_{1} \in U$ be a generator of $u^{\perp} \cap U$ and $e_{2}$ be a generator of the direct summand $(-2)$ appearing in Decomposition (1.0.7). Then

$$
\Lambda:=u^{\perp}=U^{2} \widehat{\oplus}\left(-E_{8}\right)^{2} \widehat{\oplus} \mathbb{Z} e_{1} \widehat{\oplus} \mathbb{Z} e_{2} \cong U^{2} \widehat{\oplus}\left(-E_{8}\right)^{2} \widehat{\oplus}(-2)^{2} .
$$

The period domain of interest to us is

$$
\mathcal{D}_{2}:=\{[v] \in \mathbb{P}(\Lambda \otimes \mathbb{C}) \mid(v, v)=0, \quad(v, \bar{v})>0\} .
$$

Let $\operatorname{Stab}(u)<O(\widetilde{\Lambda})$ be the subgroup of isometries fixing $u$ and $\rho: \operatorname{Stab}(u) \rightarrow$ $O(\Lambda)$ be the restriction map - this makes sense because $\Lambda=u^{\perp}$. Let

$$
\Gamma:=\operatorname{Im}(\rho)<O(\Lambda) .
$$

The period moduli space is the quotient

$$
\mathcal{Q}_{2}:=\Gamma \backslash \mathcal{D}_{2} .
$$


We will prove in Subsection (2.2) that

$$
[O(\Lambda): \Gamma]=2 .
$$

An explicit $r \in(O(\Lambda) \backslash \Gamma)$ is the isometry which interchanges $e_{1}$ with $e_{2}$ and fixes the elements of $\left\{e_{1}, e_{2}\right\}^{\perp}$, see (2.2.12). By (1.0.13) the involution $r$ descends to a non-trivial involution

$$
\bar{r}: \mathcal{Q}_{2} \rightarrow \mathcal{Q}_{2}
$$

Let $A \in \mathbb{L} \mathbb{G}\left(\wedge^{3} V\right)^{0}$ : the isomorphism class of the Hodge structure on $c_{1}\left(L_{A}\right)^{\perp} \subset$ $H^{2}\left(X_{A}\right)$ is a point of $\mathcal{Q}_{2}$ (see Section (2)) that we denote by $\mathcal{P}(A)$ - this is the period point of $X_{A}$. The global period map is defined by

$$
\begin{array}{cc}
\mathbb{L} \mathbb{G}\left(\wedge^{3} V\right)^{0} & \stackrel{\mathcal{P}}{\longrightarrow} \mathcal{Q}_{2} \\
A & \mapsto \mathcal{P}(A) .
\end{array}
$$

Of course we also have a global period map $\mathbb{L} \mathbb{G}\left(\wedge^{3} V^{\vee}\right)^{0} \rightarrow \mathcal{Q}_{2}$; we denote it by the same symbol $\mathcal{P}$. If $A \in \mathbb{L} \mathbb{G}\left(\wedge^{3} V\right)^{00}$ we have two period points namely $\mathcal{P}(A)$ and $\mathcal{P}\left(A^{\perp}\right)$. The following is the main result of this paper.

Theorem 1.1. Keep notation as above. If $A \in \mathbb{L} \mathbb{G}\left(\wedge^{3} V\right)^{00}$ then

$$
\mathcal{P}\left(A^{\perp}\right)=\bar{r} \circ \mathcal{P}(A) .
$$

The theorem may be stated informally as follows: the polarized Hodge structures on $c_{1}\left(L_{A}\right)^{\perp} \subset H^{2}\left(X_{A}\right)$ and $c_{1}\left(L_{A^{\perp}}\right)^{\perp} \subset H^{2}\left(X_{A^{\perp}}\right)$ (orthogonality is with respect to the Beauville-Bogomolov quadratic form) are isomorphic while in general there is no Hodge isometry between $H^{2}\left(X_{A}\right)$ and $H^{2}\left(X_{A^{\perp}}\right)$. Theorem (1.1) naturally raises the following question: are $X_{A}$ and $X_{A^{\perp}}$ Fourier-Mukai partners ? We expand on this. Let $T\left(X_{A}\right):=H_{\mathbb{Z}}^{1,1}\left(X_{A}\right)^{\perp} \subset H^{2}\left(X_{A} ; \mathbb{Z}\right)$ and $T\left(X_{A^{\perp}}\right):=H_{\mathbb{Z}}^{1,1}\left(X_{A^{\perp}}\right)^{\perp} \subset H^{2}\left(X_{A^{\perp}} ; \mathbb{Z}\right)$ be the transcendental lattices of $X_{A}$ and $X_{A^{\perp}}$ respectively. By Theorem (1.1) the polarized Hodge structures on $T\left(X_{A}\right) \otimes \mathbb{C}$ and $T\left(X_{A^{\perp}}\right) \otimes \mathbb{C}$ are isomorphic: by analogy with the Orlov-Mukai Theorem on $K 3$ surfaces one may wonder whether this implies that the derived categories of bounded complexes of coherent sheaves on $X_{A}$ and $X_{A^{\perp}}$ are equivalent, i.e. $X_{A}$ and $X_{A^{\perp}}$ are Fourier-Mukai partners.

There are three steps in the proof of the above theorem. First of all there exists a rational Hodge isometry

$$
H^{2}\left(X_{A}\right) \supset c_{1}\left(L_{A}\right)^{\perp} \cong c_{1}\left(L_{A^{\perp}}\right)^{\perp} \subset H^{2}\left(X_{A^{\perp}}\right) .
$$


In fact let $\widetilde{Y}_{A}, \widetilde{Y}_{A^{\perp}}$ be the desingularizations of $Y_{A}, Y_{A^{\perp}}$ respectively - they are obtained by blowing up $\operatorname{sing}\left(Y_{A}\right)$ and $\operatorname{sing}\left(Y_{A^{\perp}}\right)$ respectively. From the equality $Y_{A}^{\vee}=Y_{A^{\perp}}$ one gets that

$$
\widetilde{Y}_{A} \cong \widetilde{Y}_{A^{\perp}}
$$

Now $H^{4}\left(Y_{A}\right)$ is a subgroup of $\operatorname{Sym}^{2} H^{2}\left(X_{A}\right)$ and similarly for $H^{4}\left(Y_{A^{\perp}}\right)$; this fact together with (1.0.18) gives (1.0.17) after some work. It follows from (1.0.17) that locally (in the classical topology) there exists $g \in O(\Lambda \otimes \mathbb{Q}$ ) relating the periods of $X_{A}$ and $X_{A^{\perp}}$; this means that we have

$$
\mathcal{P}_{\Phi}\left(A^{\perp}\right)=g \circ \mathcal{P}_{\Psi}(A)
$$

where $\mathcal{P}_{\Psi}, \mathcal{P}_{\Phi}$ are local liftings of the global period maps ( $\Psi, \Phi$ are markings of the weight-2 cohomology). The second step consists in showing that (1.0.16) holds for $A$ belonging to a certain locally closed codimension-1 submanifold $\Delta_{*}^{0}(V) \subset$ $\mathbb{L} \mathbb{G}\left(\wedge^{3} V\right)^{0}$. If $A \in \Delta_{*}^{0}(V)$ then $X_{A}$ is isomorphic to a moduli space of sheaves on a $K 3$ surface $S \subset \mathbb{P}^{6}$ of degree 10 . On the other hand there is a map $f: S^{[2]} \rightarrow$ $Y_{A^{\perp}}$ of degree 2 which contracts a certain plane $P \subset S^{[2]}$ and is finite over $Y_{A^{\perp}} \backslash f(P)$. One shows that mult $_{f(P)} Y_{A^{\perp}}=3$ and hence $A^{\perp} \notin \mathbb{L} \mathbb{G}\left(\wedge^{3} V^{\vee}\right)^{0}$ i.e. $A \notin \mathbb{L} \mathbb{G}\left(\wedge^{3} V\right)^{00}$. Morally $X_{A^{\perp}}$ is the singular symplectic 4 -fold obtained from $S^{[2]}$ by contracting the plane $P$. We do not prove a precise version of this; however we do prove that the local period map of $\mathbb{L} \mathbb{G}\left(\wedge^{3} V^{\vee}\right)^{0}$ extends across $A^{\perp}$ and that its value is given by the periods of $S^{[2]}$. This will allow us to check that (1.0.16) holds for $A \in \Delta_{*}^{0}(V)$. Since the local period map sends $\Delta_{*}^{0}(V)$ to the intersection of $\mathcal{D}_{2}$ and a hyperplane $\zeta^{\perp}$ it will follow that the rational isometry $g$ of (1.0.19) is (with suitable markings $\Psi, \Phi)$ either $r$ or $r \circ r_{\zeta}$ where $r_{\zeta}$ is the reflection in the span of $\zeta$. We rule out this second possibility by a monodromy argument: this is the third step in the proof of Theorem (1.1).

The paper is organized as follows. In Section (2) we recall the definition of local and global period maps and we prove (1.0.13). In the next section we prove the results on $\Delta_{*}^{0}(V)$ that we described above. In the final section we prove the existence of a rational Hodge isometry (1.0.17) and we give the monodromy argument.

Notation: $V$ will always be a complex vector-space of dimension 6 . 


\section{The PeRIOD MAP AND THE LATtice $\Lambda$}

2.1. The period map. Let $X$ be a deformation of $(K 3)^{[2]}$. Let $(\cdot, \cdot)_{X}$ be the Beauville-Bogomolov symmetric bilinear form on $H^{2}(X)$. The restriction of $(\cdot, \cdot)_{X}$ to $H^{2}(X ; \mathbb{Z})$ is a non-degenerate integral symmetric form; thus $H^{2}(X ; \mathbb{Z})$ is a lattice. As is well-known $H^{2}(X ; \mathbb{Z})$ and $\widetilde{\Lambda}$ are isometric. Now assume that we are given a holomorphic line-bundle $L$ on $X$ such that $\left(c_{1}(L), c_{1}(L)\right)_{X}=2$. Since $O(\widetilde{\Lambda})$ acts transitively on the set of vectors of square 2 there exists an isometry

$$
\psi: H^{2}(X ; \mathbb{Z}) \stackrel{\sim}{\longrightarrow} \widetilde{\Lambda}
$$

such that $\psi\left(c_{1}(L)\right)=u$ where $u$ is as in (1.0.8); this is a marking of $(X, L)$. Let $\psi_{\mathbb{C}}: H^{2}(X ; \mathbb{C}) \stackrel{\sim}{\longrightarrow} \widetilde{\Lambda} \otimes \mathbb{C}$ be the map obtained from $\psi$ by extension of scalars. Let $\sigma$ be a generator of $H^{2,0}(X)$; then

$$
\left(c_{1}(L), \sigma\right)_{X}=0=(\sigma, \sigma)_{X}, \quad(\sigma, \bar{\sigma})_{X}>0 .
$$

Thus

$$
\mathcal{P}_{\psi}(X, L):=\psi_{\mathbb{C}}\left(H^{2,0}(X)\right) \in \mathcal{D}_{2} .
$$

This is the local period point of $(X, L)$ associated to $\psi$ (or periods of $(X, L)$ ). Any other marking of $(X, L)$ is given by $\gamma \circ \psi$ where $\gamma \in \Gamma$, thus the $\Gamma$-orbit of $\mathcal{P}_{\psi}(X, L)$ is a well-defined point of $\mathcal{Q}_{2}$; this is the global period point of $(X, L)$, we denote it by $\mathcal{P}(X, L)$. If $A \in \mathbb{L} \mathbb{G}\left(\wedge^{3} V\right)^{0}$ we let $\mathcal{P}(A):=\mathcal{P}\left(X_{A}, L_{A}\right)$.

Later on we will study global and local period maps for certain families of double EPW-sextics. Let

$$
\pi: \mathcal{X} \rightarrow T
$$

be a proper submersive map between complex manifolds such that each fiber is a deformation of $(K 3)^{[2]}$. For $t \in T$ we let $X_{t}:=\pi^{-1}(t)$. We assume that we are given a (holomorphic) line-bundle $\mathcal{L}$ on $\mathcal{X}$ such that $c_{1}\left(\left.\mathcal{L}\right|_{X_{t}}\right)$ has square 2 for every $t \in T$; we let $L_{t}:=\left.\mathcal{L}\right|_{X_{t}}$. The global period map of $(\mathcal{X}, \mathcal{L})$ is given by

$$
\begin{array}{ll}
T \stackrel{\mathcal{P}}{\longrightarrow} & \quad \mathcal{Q}_{2} \\
t & \mapsto \mathcal{P}(t):=\mathcal{P}\left(X_{t}, L_{t}\right)
\end{array}
$$

Griffiths proved that $\mathcal{P}$ is a holomorphic map. Let $\pi: \mathcal{X} \rightarrow \mathbb{L} \mathbb{G}\left(\wedge^{3} V\right)^{0}$ be the tautological family of double EPW-sextics and $\mathcal{L}$ be the tautological relatively ample line-bundle on $\mathcal{X}$ which restricts to $L_{A}$ (see (1.0.3)) on $X_{A}$; then $\mathcal{P}$ is the 
period map of (1.0.15), in particular the map of (1.0.15) is holomorphic. Let's go back to a general family (2.1.4): it is not always possible to lift the global period map $\mathcal{P}$ to a map $T \rightarrow \mathcal{D}_{2}$, in fact a necessary and sufficient condition is that $R^{2} \pi_{*} \mathbb{Z}$ is trivial. Suppose that $R^{2} \pi_{*} \mathbb{Z}$ is trivial. Then there exists a trivialization $\Psi: R^{2} \pi_{*} \mathbb{Z} \stackrel{\sim}{\longrightarrow} T \times \widetilde{\Lambda}$ sending the section corresponding to $c_{1}(\mathcal{L})$ to the section given by $u-$ this is a marking of $(\mathcal{X}, \mathcal{L})$. Given such a marking we let $\Psi_{t}: H^{2}\left(X_{t} ; \mathbb{Z}\right) \stackrel{\sim}{\longrightarrow} \widetilde{\Lambda}$ be the fiber of $\Psi$ over $t$. The local period map of $(\mathcal{X}, \mathcal{L})$ associated to $\Psi$ is given by

$$
\begin{aligned}
T \stackrel{\mathcal{P}_{\Psi}}{\longrightarrow} & \mathcal{D}_{2} \\
t \stackrel{P_{\Psi}}{\longmapsto}(t):= & \mathcal{P}_{\Psi_{t}}\left(X_{t}, L_{t}\right) .
\end{aligned}
$$

Griffiths proved that $\mathcal{P}_{\Psi}$ is holomorphic.

2.2. Proof of (1.0.13). We start by recalling the definition of discriminant group and discriminant quadratic form of an even lattice $\left(L,(\cdot, \cdot)_{L}\right)$, i.e. a free finitely generated abelian group $L$ equipped with a symmetric integral even nondegenerate bilinear form $(\cdot, \cdot)_{L}$. We follow [10]. The bilinear form $(\cdot, \cdot)_{L}$ extends to a $\mathbb{Q}$-valued bilinear form on $L \otimes \mathbb{Q}$; abusing notation we denote by $(\cdot, \cdot)_{L}$ the extended form. Let $L^{\vee}:=\operatorname{Hom}(L, \mathbb{Z})$; by non-degeneracy of $(\cdot, \cdot)_{L}$ we have a natural chain of inclusions

$$
L \subset L^{\vee} \subset L \otimes \mathbb{Q}
$$

The discriminant group of $L$ is $A_{L}:=L^{\vee} / L$; it comes provided with the discriminant bilinear-form

$$
\begin{aligned}
& A_{L} \times A_{L} \stackrel{b_{L}}{\longrightarrow} \mathbb{Q} / \mathbb{Z} \\
& ([\alpha],[\beta]) \mapsto\left[(\alpha, \beta)_{L}\right]
\end{aligned}
$$

and the discriminant quadratic-form

$$
\begin{aligned}
& A_{L} \stackrel{q_{L}}{\longrightarrow} \mathbb{Q} / 2 \mathbb{Z} \\
& {[\alpha] \mapsto\left[(\alpha, \alpha)_{L}\right] .}
\end{aligned}
$$

The formula

$$
q_{L}([\alpha+\beta]) \equiv q_{L}([\alpha])+q_{L}([\beta])+2 b_{L}([\alpha],[\beta]) \quad(\bmod 2 \mathbb{Z})
$$

shows that $q_{L}$ determines uniquely $b_{L}$. An index-i overlattice of $L$ consists of a lattice $M$ and an inclusion of lattices $L \subset M$ (the restriction of $(\cdot, \cdot)_{M}$ to $L$ is equal to $\left.(\cdot, \cdot)_{L}\right)$ of index $i$. Two overlattices $M_{1} \supset L \subset M_{2}$ are equivalent if there 
exists an isometry $M_{1} \stackrel{\sim}{\longrightarrow} M_{2}$ which is the identity on $L$, i.e. if the inclusions $M_{i} \hookrightarrow L^{\vee}$ for $i=1,2$ have the same image. Suppose that $L \subset M$ is an index- $i$ overlattice of $L$; the inclusion $M \subset L^{\vee}$ defines an inclusion $M / L \subset A_{L}$ with image a subgroup of cardinality $i$ which is $q_{L}$-isotropic. This construction defines a one-to-one correspondence between the set of equivalence classes of indexi- $i$ overlattices of $L$ and the set of $q_{L}$-isotropic subgroups of $A_{L}$ of cardinality $i$. The correspondence is equivariant for the natural actions of $O(L)$ on both sets. Now consider the lattice $L=\mathbb{Z} u \widehat{\oplus} \Lambda$ where $u$ and $\Lambda$ are as in Section (1): we will describe the discriminant group and discriminant form of $L$. Let $e_{1}, e_{2} \in \Lambda$ be as in Section (1). A straightforward computation gives that

$$
A_{L}=\mathbb{Z} /(2)\left[\frac{u}{2}\right] \oplus \mathbb{Z} /(2)\left[\frac{e_{1}}{2}\right] \oplus \mathbb{Z} /(2)\left[\frac{e_{2}}{2}\right]
$$

and that

$$
q_{L}\left(x\left[\frac{u}{2}\right]+y_{1}\left[\frac{e_{1}}{2}\right]+y_{2}\left[\frac{e_{2}}{2}\right]\right) \equiv \frac{1}{2}\left(x^{2}-y_{1}^{2}-y_{2}^{2}\right) \quad(\bmod 2 \mathbb{Z}) .
$$

The set $I \subset A_{L}$ of non-zero isotropic vectors is given by

$$
I=\left\{\left[\frac{u}{2}\right]+\left[\frac{e_{1}}{2}\right],\left[\frac{u}{2}\right]+\left[\frac{e_{2}}{2}\right]\right\} .
$$

The group $O(L)$ acts naturally on $A_{L}$ and hence also on $I$; thus we have a homomorphism

$$
O(L) \stackrel{\epsilon}{\longrightarrow} A u t(I) \cong \mathbb{Z} /(2) .
$$

The overlattice $\widetilde{\Lambda} \supset L$ is of index 2 because $L$ is the kernel of the surjection

$$
\begin{aligned}
& \widetilde{\Lambda} \longrightarrow \quad \mathbb{Z} /(2) \\
& v \mapsto(v, u) \quad(\bmod 2) .
\end{aligned}
$$

The correspondence described above defines an $O(L)$-equivariant bijective map between $I$ and the set of equivalence classes of index-2 overlattices of $L$; thus

$$
\operatorname{Im}(O(\widetilde{\Lambda}) \rightarrow O(L))=\operatorname{ker}(\epsilon) .
$$

The subgroup of $O(L)$ consisting of isometries which are the identity on $\mathbb{Z} u$ is naturally identified with $O(\Lambda)$; thus $O(\Lambda)<O(L)$. By (2.2.10) and the definition of $\Gamma$ (see (1.0.11)) we get that

$$
\Gamma=\operatorname{ker}\left(\left.\epsilon\right|_{O(\Lambda)}\right)
$$


Let $r \in O(\Lambda)$ be the involution characterized by the following properties:

$$
r\left(e_{1}\right)=e_{2}, \quad r\left(e_{2}\right)=e_{1},\left.\quad r\right|_{\left\{e_{1}, e_{2}\right\}^{\perp}}=\text { identity. }
$$

Then $\epsilon(r)$ is the non-trivial permutation of $I$ and hence $\left.\epsilon\right|_{O(\Lambda)}$ is surjective. Thus (1.0.13) follows from (2.2.11).

\section{Explicit DUAL COUPLES}

Let $F_{5}^{3} \subset \mathbb{P}^{6}$ be the intersection of $G r\left(2, \mathbb{C}^{5}\right) \subset \mathbb{P}\left(\wedge^{2} \mathbb{C}^{5}\right) \cong \mathbb{P}^{9}$ with a transversal 6-dimensional linear subspace of $\mathbb{P}^{9}$. Let $T \subset\left|\mathcal{O}_{\mathbb{P}^{6}}(2)\right|$ be the open dense subset parametrizing quadrics which are transversal to $F_{5}^{3}$. For $t \in T$ let $Q_{t}$ be the quadric corresponding to $t$ and

$$
S_{t}:=F_{5}^{3} \cap Q_{t} .
$$

Then $S_{t}$ is a degree-10 linearly normal $K 3$ surface; in fact the generic such surface is projectively equivalent to $S_{t}$ for some $t \in T$ by Mukai [8]. Let $D_{t}$ be the hyperplane divisor class on $S_{t}$ and $M_{t}$ be the moduli space of $D_{t}$-semistable rank-2 sheaves $\mathcal{F}$ on $S_{t}$ with $c_{1}(\mathcal{F})=c_{1}\left(D_{t}\right)$ and $c_{2}(\mathcal{F})=5$. Suppose that

$$
\text { for all divisors } E \text { on } S_{t} \text { we have } E \cdot D_{t} \equiv 0 \quad(\bmod 10) \text {. }
$$

Then (see Section (5) of [13]) there exists $A_{t} \in \mathbb{L} \mathbb{G}\left(\wedge^{3} V\right)^{0}$ such that

$$
X_{A_{t}} \cong M_{t} .
$$

Furthermore $Y_{A_{t}}$ is explicitely described as follows. Let $\Sigma_{t}$ be the divisor on $\left|I_{S_{t}}(2)\right|$ parametrizing singular quadrics, since all quadrics parametrized by $\left|I_{F_{5}^{3}}(2)\right|$ are singular we have

$$
\Sigma_{t}=\left|I_{F_{5}^{3}}(2)\right|+\Sigma_{t}^{\prime}
$$

Then

$$
Y_{A_{t}} \cong \Sigma_{t}^{\prime}
$$

(Formally $\Sigma_{t}^{\prime}$ is a degree-6 divisor; in the above equation we are implicitely stating that $\Sigma_{t}^{\prime}$ is a reduced divisor and hence we may view it as a degree-6 hypersurface.) The set of $t$ for which (3.0.2) holds is the complement of a countable union of proper algebraic subvarieties of $T$ however a straightforward argument shows that there is an open dense $T^{\prime \prime \prime} \subset T$ such that for $t \in T^{\prime \prime \prime}$ there exists $A_{t} \in \mathbb{L} \mathbb{G}\left(\wedge^{3} V\right)^{0}$ for which both (3.0.3) and (3.0.5) hold; we give the argument in Subsection (3.1). 
We let $\Delta^{0}(V) \subset \mathbb{L} \mathbb{G}\left(\wedge^{3} V\right)^{0}$ be the set of $A$ such that $X_{A}$ is isomorphic to $M_{t}$ for some $t \in T^{\prime \prime \prime} ;$ this is a locally closed subset of $\mathbb{L} \mathbb{G}\left(\wedge^{3} V\right)^{0}$. Computing the periods of $X_{A}$ for $A \in \Delta^{0}(V)$ we will show that $\Delta^{0}(V)$ has codimension 1 in $\mathbb{L} \mathbb{G}\left(\wedge^{3} V\right)^{0}$. Let $A \in \Delta^{0}(V)$, thus $X_{A} \cong M_{t}$ for some $t \in T$ : the dual $Y_{A}^{\vee}$ is described as follows. The Hilbert scheme $S_{t}^{[2]}$ contains a copy of $\mathbb{P}^{2}$, call it $P_{t}$, parametrizing $Z \subset S_{t}$ which span a line contained in $F_{5}^{3}$. Let $S_{t}^{[2]} \rightarrow N_{t}$ be the contraction of $P_{t}$ - thus $N_{t}$ is a singular symplectic variety. There is an involution on $N_{t}$ whose quotient is isomorphic to $Y_{A}^{\vee}$. From this it will follow that $A \notin \mathbb{L} \mathbb{G}\left(\wedge^{3} V\right)^{00}$ and hence apparently it will not make sense to compute $\mathcal{P}\left(A^{\perp}\right)$. The main point of this subsection is to prove that the period map extends across $A^{\perp}$, in fact the local period map extends and its value at $A^{\perp}$ is given by the periods of $\left(S_{t}^{[2]}, D_{t}\right)$.

3.1. The locus $\Delta^{0}(V)$. We recall that the Mukai vector $v(\mathcal{F})$ of a sheaf $\mathcal{F}$ on $S_{t}$ is

$$
v(\mathcal{F}):=\operatorname{ch}(\mathcal{F}) \sqrt{\operatorname{Td}\left(S_{t}\right)}=\operatorname{ch}(\mathcal{F})\left(1+\eta_{t}\right) \in H^{*}\left(S_{t} ; \mathbb{Z}\right),
$$

where $\eta_{t} \in H^{4}\left(S_{t} ; \mathbb{Z}\right)$ is the orientation class. If $[\mathcal{F}] \in M_{t}$ (we let $[\mathcal{F}] \in M_{t}$ be the equivalence class of the semistable sheaf $\mathcal{F}$ - we recall that if $\mathcal{F}$ is stable this is the same as the isomorphism class of $\mathcal{F}$ ) the class $v(\mathcal{F})$ is independent of $\mathcal{F}$, we denote it by $v_{t}$; explicitly

$$
v_{t}=2+c_{1}\left(D_{t}\right)+2 \eta_{t} .
$$

Proposition 3.1. Keep notation as above. There is an open dense subset $T^{\prime \prime \prime} \subset$ $T$ such that the following holds. Let $t \in T^{\prime \prime \prime} ;$ then there exists $A_{t} \in \mathbb{L} \mathbb{G}\left(\wedge^{3} V\right)^{0}$ such that both (3.0.3) and (3.0.5) hold. In particular we have a canonical identification $\mathbb{P}(V) \cong\left|I_{S_{t}}(2)\right|$.

Proof. By Maruyama [7] there exists a projective map $\rho: \mathcal{M} \rightarrow T$ with (schematic) fiber $M_{t}$ over $t \in T$. Let $t \in T$; we say that $S_{t}$ is unsuitable if there exists a divisor class $C$ on $S_{t}$ such that

$$
C \cdot D_{t}=0, \quad-10 \leq C \cdot C<0 .
$$

The set of unsuitable $t$ is a proper closed subset of $T$, thus the complement $T^{\prime}$ is an open dense subset of $T$. Let $\mathcal{M}^{\prime}:=\rho^{-1}\left(T^{\prime}\right)$ and $\rho^{\prime}: \mathcal{M}^{\prime} \rightarrow T^{\prime}$ be the restriction of $\rho$. It is known that if $t \in T^{\prime}$ then every sheaf parametrized by $M_{t}$ is slope-stable and $M_{t}$ is a smooth 4 -dimensional scheme (Main Theorem (0.1.2) 
and Proposition (2.1) of [11] - notice that $t \in T^{\prime}$ if and only if $D_{t}$ is $\left|v_{t}\right|$-generic). Let's show that there is an open dense $T^{\prime \prime} \subset T^{\prime}$ such that for $t \in T^{\prime \prime}$ the Mukai reflection (see (4.2.2) of [12]) is a regular involution $\phi_{t}$ on $M_{t}$ - we recall that in general the Mukai reflection acts on the derived category of coherent sheaves on $S_{t}$. In order for $\phi_{t}$ to be a regular involution on $M_{t}$ it suffices that for all $[\mathcal{F}] \in M_{t}$ the following hold:

(a) $h^{0}(\mathcal{F})=\chi(\mathcal{F})=4$,

(b) $\mathcal{F}$ is globally generated away from (at most) a zero-dimensional subset of $S_{t}$,

(c) the kernel of the evaluation map $H^{0}(\mathcal{F}) \otimes \mathcal{O}_{S_{t}} \rightarrow \mathcal{F}$, call it $\mathcal{E}$, is a $D_{t^{-}}$ slope-stable sheaf.

If (a)-(b)-(c) are satisfied for all $[\mathcal{F}] \in M_{t}$ then the generic sheaf $\mathcal{F}$ parametrized by $M_{t}$ is globally generated and for such a sheaf $\phi_{t}([\mathcal{F}])=\left[\mathcal{E}^{\vee}\right]$ where $\mathcal{E}$ is as in (c) above. Let $T^{\prime \prime} \subset T^{\prime}$ be the set of $t$ such that (a)-(b)-(c) hold for every $[\mathcal{F}] \in M_{t}$. Let's prove that $T^{\prime \prime}$ is open. First we show that the subset $T_{a} \subset T$ of $t \in T^{\prime}$ such that (a) holds for every $[\mathcal{F}] \in M_{t}$ is open. Let $t \in T^{\prime}$ : if $[\mathcal{F}] \in M_{t}$ then by stability $h^{2}(\mathcal{F})=0$ and hence $h^{0}(\mathcal{F}) \geq 4$, thus by the upper-semicontinuity Theorem the set of $[\mathcal{F}] \in M_{t}$ that violate (a) is closed. By properness of the map $\rho^{\prime}: \mathcal{M}^{\prime} \rightarrow T^{\prime}$ it follows that $\left(T^{\prime} \backslash T_{a}\right)$ is closed i.e. $T_{a}$ is open. A similar argument shows that the set of $t \in T_{a}$ such that (b)-(c) hold for every $[\mathcal{F}] \in M_{t}$ is open; thus $T^{\prime \prime}$ is open. By Lemmas (4.10)-(4.11) of [12] we know that $T^{\prime \prime}$ contains the subset of $t$ such that (3.0.2) holds; since the latter set is dense in $T$ we get that $T^{\prime \prime}$ is dense in $T$. Let $T^{\prime \prime \prime} \subset T^{\prime \prime}$ be the set of $t$ such that

$$
S_{t} \text { contains no effective non-zero divisor } E \text { with } E \cdot D_{t} \leq 5 \text {. }
$$

Thus $T^{\prime \prime \prime}$ is open and dense in $T$. We claim that if $t \in T^{\prime \prime \prime}$ then

$$
M_{t} /\left\langle\phi_{t}\right\rangle \cong \Sigma_{t}^{\prime} \subset\left|I_{S_{t}}(2)\right| \cong \mathbb{P}^{5}
$$

More precisely Proposition (5.1) of [12] holds for $M_{t}=\mathcal{M}\left(v_{t}\right)$. In order to prove this it suffices to show that for every $t \in T^{\prime \prime \prime}$ the following holds:

(1) If $[\mathcal{F}] \in M_{t}$ and $\sigma \in H^{0}(\mathcal{F})=\operatorname{Hom}\left(\mathcal{O}_{S_{t}}, \mathcal{F}\right)$ is non-zero then the quotient $\mathcal{F} / \operatorname{Im}(\sigma)$ is locally-free in codimension 1 ( $\sigma$ has isolated zeroes) - this is used in the proof of Lemma (5.4) of [12]. 
(2) If $\mathcal{G}$ is a globally generated rank-2 vector bundle on $S_{t}$ with $\operatorname{det} \mathcal{G} \cong$ $\mathcal{O}_{S_{t}}\left(D_{t}\right)$ and $c_{2}(\mathcal{G})=5$ then $\mathcal{G}$ is $D_{t}$-slope stable - this is used in the proof of Lemma (5.7) of [12].

Let's show that (1) above holds. Suppose that $\sigma$ does not have isolated zeroes: then it vanishes along a non-zero effective divisor $E$ and by slope stability of $\mathcal{F}$ we have $E \cdot D_{t}<\operatorname{slope}(\mathcal{F})=5$ contradicting (3.1.4). Let's show that (2) above holds. Suppose that $\mathcal{G}$ is not $D_{t}$-slope stable; since $\mathcal{G}$ has rank 2 there is a destabilizing sequence $\mathcal{G} \rightarrow I_{Z} \otimes \mathcal{O}_{S_{t}}(E)$ where $Z \subset S_{t}$ is zero-dimensional and $E$ is a divisor with $E \cdot D_{t} \leq \operatorname{slope}(\mathcal{F})=5$. Since $\mathcal{G}$ is globally generated there is a non-zero section of $I_{Z} \otimes \mathcal{O}_{S_{t}}(E)$ and hence $E$ is effective; by (3.1.4) we get that $E=0$. Thus we have an exact sequence

$$
0 \longrightarrow \mathcal{O}_{S_{t}}\left(D_{t}\right) \longrightarrow \mathcal{G} \longrightarrow I_{Z} \longrightarrow 0
$$

Since $\ell(Z)=c_{2}(\mathcal{G})=5$ the zero-dimensional subscheme $Z \subset S_{t}$ is non-empty and hence $h^{0}\left(I_{Z}\right)=0$; this contradicts the hypothesis that $\mathcal{G}$ is globally generated. We have proved that (3.1.5) holds for $t \in T^{\prime \prime \prime}$. By Theorem (1.1) of [13] we get that there exists $A_{t} \in \mathbb{L} \mathbb{G}\left(\wedge^{3} V\right)^{0}$ such that both (3.0.3) and (3.0.5) hold.

The proof of the above proposition together with Claim (5.18) of [12] gives the following result.

Corollary 3.2. Let $t \in T^{\prime \prime \prime}$ and let $A_{t} \in \mathbb{L} \mathbb{G}\left(\wedge^{3} V\right)^{0}$ be such that both (3.0.3) and (3.0.5) hold. The map $f_{A_{t}}: X_{A_{t}} \rightarrow Y_{A_{t}}$ is identified with the quotient map $M_{t} \rightarrow M_{t} /\left\langle\phi_{t}\right\rangle$.

Definition 3.3. Let $\Delta^{0}(V) \subset \mathbb{L} \mathbb{G}\left(\wedge^{3} V\right)^{0}$ be the locus of $A$ such that $X_{A}$ is isomorphic to $M_{t}$ for some $t \in T^{\prime \prime \prime}$.

We will show that $\Delta^{0}(V)$ is a locally closed subset of codimension 1 . First we recall how one describes $H^{2}\left(M_{t}\right)$ for $t \in T^{\prime}$. Let $u, w \in H^{*}\left(S_{t}\right)$ and let $u_{q}, w_{q}$ be the degree- $q$ components of $u, w$ respectively. One sets $u^{\vee}:=u_{0}-u_{2}+u_{4}$ and

$$
\langle u, w\rangle:=\int_{S_{t}}\left(u_{2} \wedge w_{2}-u_{0} w_{4}-u_{4} w_{0}\right)=-\int_{S_{t}} u^{\vee} \wedge w .
$$


This is Mukai's bilinear symmetric form. One defines a positive ${ }^{2}$ weight-two Hodge structure on $H^{*}\left(S_{t}\right)$ by defining the Hodge filtration as

$$
F^{1} H^{*}\left(S_{t}\right):=H^{0}\left(S_{t}\right) \oplus F^{1} H^{2}\left(S_{t}\right) \oplus H^{4}\left(S_{t}\right), \quad F^{2} H^{*}\left(S_{t}\right):=F^{2} H^{2}\left(S_{t}\right) .
$$

Let $v_{t}$ be the Mukai vector $(3.1 .2)$, then $v_{t}$ is integral of type $(1,1)$ and hence $v_{t}^{\perp}$ is an integral Hodge substructure of $H^{*}\left(S_{t}\right)$ and furthermore the restriction of Mukai's bilinear symmetric form to $v_{t}^{\perp}$ is integral. Mukai defined (see $\left.[9,11]\right)$ a map

$$
\theta_{t}: v_{t}^{\perp} \longrightarrow H^{2}\left(M_{t}\right)
$$

by taking Künneth components of the Chern character of a tautological sheaf on $S_{t} \times M_{t}$ (if such a sheaf does not exist one considers a quasi-tautological sheaf). In [11] we proved that $\theta_{t}$ is an isomorphism of Hodge structures and an isometry of lattices - of course the bilinear form on $v_{t}^{\perp}$ is the restriction of the Mukai pairing. Now assume that $t \in T^{\prime \prime \prime}$ and let $A_{t}$ be as in Proposition (3.1) and $L_{A_{t}}$ be as in (1.0.3); then by Corollary (3.2) and Proposition (5.1) of [12] we have

$$
c_{1}\left(L_{A_{t}}\right)=\theta_{t}\left(\eta_{t}-1\right)
$$

We let $h_{t}:=\theta_{t}\left(\eta_{t}-1\right)$.

Proposition 3.4. Keep notation as above. Then $\Delta^{0}(V)$ is a $P G L(V)$-invariant subset of $\mathbb{L} \mathbb{G}\left(\wedge^{3} V\right)^{0}$ which is locally (in the classical topology) a codimension 1 submanifold.

Proof. The subset $\Delta^{0}(V)$ is $P G L(V)$-invariant by definition. Let $\pi: \mathcal{X} \rightarrow \mathbb{L} \mathbb{G}\left(\wedge^{3} V\right)^{0}$ be the tautological family of double EPW-sextics and $\mathcal{L}$ be the tatutological relatively ample line-bundle on $\mathcal{X}$; thus the restriction of $\mathcal{L}$ to $X_{A}$ is isomorphic to $L_{A}$. Let $A_{p} \in \Delta^{0}(V)$. Thus there exists $p \in T^{\prime \prime \prime}$ such that $X_{A_{p}} \cong M_{p}$. Let $U \subset \mathbb{L} \mathbb{G}\left(\wedge^{3} V\right)^{0}$ be a small open ball contining $A_{p}$. Let $\mathcal{X}_{U}:=\pi^{-1}(U)$ and $\mathcal{L}_{U}:=\left.\mathcal{L}\right|_{\mathcal{X}_{U}}$. Since $U$ is contractible there is a marking $\Psi$ of $\left(\mathcal{X}_{U}, \mathcal{L}_{U}\right)$; let $\mathcal{P}_{\Psi}: U \rightarrow \mathcal{D}_{2}$ be the corresponding local period map. We notice that $(5+$ $\left.2 c_{1}\left(D_{p}\right)+5 \eta_{p}\right) \in v_{p}^{\perp}$ and hence $\theta_{p}\left(5+2 c_{1}\left(D_{p}\right)+5 \eta_{p}\right) \in H^{2}\left(M_{p}\right)$. Furthermore since $\left\langle\eta_{p}-1,5+2 c_{1}\left(D_{p}\right)+5 \eta_{p}\right\rangle=0$ we have $\left(h_{p}, \theta_{p}\left(5+2 c_{1}\left(D_{p}\right)+5 \eta_{p}\right)\right)_{M_{p}}=0$;

\footnotetext{
${ }^{2}$ This means that $h^{p, q}=0$ if $p<0$.
} 
since $h_{p}=c_{1}\left(L_{A_{p}}\right)$ we get that $\Psi_{p}\left(\theta_{p}\left(5+2 c_{1}\left(D_{p}\right)+5 \eta_{p}\right)\right) \in u^{\perp}$ where $u$ is our fixed vector of square 2 - see (1.0.8). Let's prove that

$$
\mathcal{P}_{\Psi}\left(\Delta^{0}(V) \cap U\right)=\mathcal{P}_{\Psi}(U) \cap \Psi_{p} \circ \theta_{p}\left(5+2 c_{1}\left(D_{p}\right)+5 \eta_{p}\right)^{\perp} .
$$

First we prove that

$$
\mathcal{P}_{\Psi}\left(\Delta^{0}(V) \cap U\right) \subset \mathcal{P}_{\Psi}(U) \cap \Psi_{p} \circ \theta_{p}\left(5+2 c_{1}\left(D_{p}\right)+5 \eta_{p}\right)^{\perp} .
$$

Let $\mathcal{M}^{\prime \prime \prime}:=\rho^{-1}\left(T^{\prime \prime \prime}\right)$ and $\rho^{\prime \prime \prime}: \mathcal{M}^{\prime \prime \prime} \rightarrow T^{\prime \prime \prime}$ be the restriction of $\rho$. Then $\theta_{t}(5+$ $\left.2 c_{1}\left(D_{t}\right)+5 \eta_{t}\right)$ is a flat section of $R^{2} \rho_{*}^{\prime \prime \prime} \mathbb{Z}$ and for all $t \in T^{\prime \prime \prime}$ we have

$$
\left(\theta_{t}\left(5+2 c_{1}\left(D_{t}\right)+5 \eta_{t}\right), H^{2,0}\left(S_{t}\right)\right)_{M_{t}}=0
$$

because $\theta_{t}\left(5+2 c_{1}\left(D_{t}\right)+5 \eta_{t}\right) \in H^{1,1}\left(M_{t}\right)$; the above equality gives (3.1.12). Next we prove that

$$
\mathcal{P}_{\Psi}\left(\Delta^{0}(V) \cap U\right) \supset \mathcal{P}_{\Psi}(U) \cap \Psi_{p} \circ \theta_{p}\left(5+2 \theta_{p}\left(c_{1}\left(D_{p}\right)+5 \eta_{p}\right)^{\perp} .\right.
$$

First we notice that

$$
\left\{v_{p}, \eta_{p}-1,5+2 c_{1}\left(D_{p}\right)+5 \eta_{p}\right\}^{\perp}=H^{2}\left(S_{p}\right)_{\text {prim }}
$$

where the primitive cohomology $H^{2}\left(S_{p}\right)_{\text {prim }} \subset H^{2}(S)$ is the orthogonal to $c_{1}\left(D_{p}\right)$. Since $h_{p}=\theta_{p}\left(\eta_{p}-1\right)$ we get that

$$
H^{2}\left(M_{p}\right) \supset\left\{h_{p}, \theta_{p}\left(5+2 c_{1}\left(D_{p}\right)+5 \eta_{p}\right)\right\}^{\perp}=\theta_{p}\left(H^{2}\left(S_{p}\right)_{\text {prim }}\right) .
$$

Let $\mathcal{K}_{10}$ be the period space for $K 3$ surfaces with a polarization of degree 10; Equality (3.1.16) defines an isomorphism

$$
\mathcal{D}_{2} \cap \Psi_{p} \circ \theta_{p}\left(5+2 c_{1}\left(D_{p}\right)+5 \eta_{p}\right)^{\perp} \stackrel{\sim}{\longrightarrow} \mathcal{K}_{10}
$$

which is compatible with local period maps defined by the family $\rho^{\prime \prime \prime}: \mathcal{M}^{\prime \prime \prime} \rightarrow T^{\prime}$ and the family $\zeta: \mathcal{S}^{\prime \prime \prime} \rightarrow T^{\prime \prime \prime}$ with fiber $S_{t}$ over $t \in T^{\prime \prime \prime}$. Let $\mathcal{S}_{U}^{\prime \prime \prime}:=\zeta^{-1}(U)$. Since $\mathcal{S}^{\prime \prime \prime}$ contains the generic $K 3$ of degree 10 the local period map of the family $\mathcal{S}_{U}^{\prime \prime \prime} \rightarrow U$ is a submersive map from $U$ to an open ball in $\mathcal{K}_{10}$; since (3.1.17) is an isomorphism this proves (3.1.14). We also get that $\mathcal{P}_{\Psi}$ is submersive and hence in order to show that $\Delta^{0}(V) \cap U$ is a codimension 1 submanifold it suffices to prove that

$$
\mathcal{D}_{2} \cap \Psi_{p} \circ \theta_{p}\left(5+2 c_{1}\left(D_{p}\right)+5 \eta_{p}\right)^{\perp}
$$


is smooth. The period domain $\mathcal{D}_{2}$ is an open subset of the quadric of isotropic lines for the non-degenerate quadratic form $\left.()\right|_{,\Lambda \otimes \mathbb{C}}$ and hence if $\Psi_{p} \circ \theta_{p}(5+$ $\left.2 c_{1}\left(D_{p}\right)+5 \eta_{p}\right)$ is not isotropic then (3.1.18) is smooth. Since

$$
\begin{aligned}
\left(\Psi _ { p } \circ \theta _ { p } \left(5+2 c_{1}\left(D_{p}\right)\right.\right. & \left.\left.+5 \eta_{p}\right), \Psi_{p} \circ \theta_{p}\left(5+2 c_{1}\left(D_{p}\right)+5 \eta_{p}\right)\right)= \\
= & \left\langle 5+2 c_{1}\left(D_{p}\right)+5 \eta_{p}, 5+2 c_{1}\left(D_{p}\right)+5 \eta_{p}\right\rangle=-10
\end{aligned}
$$

the intersection (3.1.18) is indeed smooth.

3.2. The dual of $Y_{A}$ for $A \in \Delta^{0}(V)$. If $A \in \mathbb{L} \mathbb{G}\left(\wedge^{3} V\right)^{00}$ then $Y_{A}^{\vee}=Y_{A^{\perp}}$ by Proposition (3.1) of [13]. As we will see $\Delta^{0}(V) \cap \mathbb{L} \mathbb{G}\left(\wedge^{3} V\right)^{00}=\emptyset$ and hence in order to show that $Y_{A}^{\vee}=Y_{A^{\perp}}$ for $A \in \Delta^{0}(V)$ we need to improve on the result of [13].

Proposition 3.5. Let $A \in \mathbb{L} \mathbb{G}\left(\wedge^{3} V\right)$ and $\mathbb{P}(W) \in \mathbb{P}\left(V^{\vee}\right)$. Then $\mathbb{P}(W) \in Y_{A^{\perp}}$ if and only if

$$
\wedge^{3} W \cap A \neq \emptyset
$$

Proof. Let $\phi \in V^{\vee}$ be a linear function such that $W=\operatorname{ker}(\phi)$ : then

$$
F_{\phi}:=\left\{\phi \wedge \psi \mid \psi \in \wedge^{2} V^{\vee}\right\}=\left(\wedge^{3} W\right)^{\perp} .
$$

By definition $\mathbb{P}(W) \in Y_{A^{\perp}}$ if and only if

$$
\{0\} \neq F_{\phi} \cap A^{\perp}=\left(\wedge^{3} W+A\right)^{\perp} .
$$

Since $10=\operatorname{dim}\left(\wedge^{3} W\right)=\operatorname{dim} A$ and $\operatorname{dim}\left(\wedge^{3} V\right)=20$ we get that (3.2.3) holds if and only if (3.2.1) holds.

Corollary 3.6. Let $A \in \mathbb{L} \mathbb{G}\left(\wedge^{3} V\right)^{0}$. Then $Y_{A^{\perp}}$ is a hypersurface and $Y_{A}^{\vee}=Y_{A^{\perp}}$.

Proof. Let $\mathbb{P}(W) \in Y_{A^{\perp}}$; by Proposition (3.5) this is equivalent to (3.2.1). Let $0 \neq \alpha \in\left(\wedge^{3} W \cap A\right)$ : since $\operatorname{dim} W=5$ there exists $v \in W$ such that $\alpha$ is divisible by $v$ and hence $[v] \in Y_{A}$. We decompose $W=\mathbb{C} v \oplus W_{0}$ and write $\alpha=v \wedge w$ where $w \in \wedge^{2} W_{0}$; by Definition (2.5) of [13] the rank of $w$ is 4 , thus $W=\operatorname{span}(v, w)$. Furthermore if $\operatorname{dim}\left(F_{[v]} \cap A\right)=1$ then $Y_{A}$ is smooth at $[v]$ and $\mathbb{P}(W)$ is the projective tangent space to $Y_{A}$ at $[v]$ - see the proof of Proposition (3.1) of [13]. By Proposition (2.8) of [13] we know that $\operatorname{dim}\left(F_{[v]} \cap A\right)=1$ unless $[v] \in \operatorname{sing}\left(Y_{A}\right)$ and in this case $\operatorname{dim}\left(F_{[v]} \cap A\right)=2$. Since $\operatorname{sing}\left(Y_{A}\right)$ is a surface a straightforward 
dimension count gives that $\mathbb{P}(W) \notin Y_{A^{\perp}}$ for generic $\mathbb{P}(W) \in \mathbb{P}\left(V^{\vee}\right)$, thus $Y_{A^{\perp}}$ is a hypersurface. The same dimension count gives that the generic $\mathbb{P}(W) \in Y_{A^{\perp}}$ is tangent to $Y_{A}$ at one of its smooth points; this proves the corollary.

We will describe explicitly $Y_{A}^{\vee}=Y_{A^{\perp}}$ for $A \in \Delta^{0}(V)$; essentially we will give a refinement of Proposition (5.20) and Corollary (5.21) of [12]. Let $t \in T^{\prime \prime \prime}$ and $S_{t}$ be the $K 3$ surface corresponding to $t$; in order to simplify notation we temporarily drop the subscript $t$. Let $R$ be the Fano variety of lines on $F_{5}^{3}$. If $[\ell] \in R$ then $\ell \not \subset S$ by (3.1.4) and hence $\ell \cap Q$ is a 0 -dimensional scheme of length 2 contained in $S$ : thus we have a regular map

$$
\begin{aligned}
& R \longrightarrow S^{[2]} \\
& \ell \mapsto \ell \cap Q .
\end{aligned}
$$

Let $P \subset S_{t}^{[2]}$ be the image of the above map: then (3.2.4) defines a regular map $R \rightarrow P$ with inverse given by

$$
\begin{gathered}
P \longrightarrow \quad R \\
{[Z] \mapsto \operatorname{span}(Z)}
\end{gathered}
$$

and hence $P$ is isomorphic to $R$. It is known [5] that $R \cong \mathbb{P}^{2}$, thus $P \cong \mathbb{P}^{2}$. Since $S^{[2]}$ is a symplectic variety it follows that we can contract $P$ :

$$
c: S^{[2]} \rightarrow N \text {. }
$$

A priori $N$ is a complex space, we will show soon that it is projective. Let $p:=c(P)$; thus $p$ is the unique singular point of $N$. On $S^{[2]}$ there is an interesting map to $\left|I_{S}(2)\right|^{\vee}$, see (4.3) of [12]; we recall the definition. The $K 3$ surface $S$ is cut out by quadrics and it contains no lines by (3.1.4); thus we have a regular map

$$
\begin{gathered}
\left.S^{[2]} \longrightarrow I_{S}(2)\right|^{\vee} \cong \mathbb{P}^{5} \\
{[Z] \mapsto\left\{Q_{\lambda} \in\left|I_{S}(2)\right| \mid \operatorname{span}(Z) \subset Q_{\lambda}\right\} .}
\end{gathered}
$$

Let $W \subset\left|I_{S}(2)\right|^{\vee}$ be the image of the above map; thus (3.2.7) defines a map $f: S^{[2]} \rightarrow W$. If $[Z] \in P$ then $f([Z])=\left|I_{F_{5}^{3}}(2)\right|$ hence $f$ is constant on $P$; we will see that the point

$$
f(P)=\left|I_{F_{5}^{3}}(2)\right| \in W
$$


is quite special. Since $f$ is constant on $P$ and $N$ is normal the map $f$ descends to a regular map

$$
\bar{f}: N \rightarrow W .
$$

Lemma 3.7. Keep notation as above. There exist a non trivial involution $\bar{\phi}: N \rightarrow$ $N$ and a birational morphism $\epsilon: N /\langle\bar{\phi}\rangle \rightarrow W$ with finite fibers such that $\bar{f}$ is the composition

$$
N \stackrel{\pi}{\longrightarrow} N /\langle\bar{\phi}\rangle \stackrel{\epsilon}{\longrightarrow} W .
$$

In particular $\operatorname{deg} \bar{f}=2, N$ is projective and $\operatorname{deg} W=6$.

Proof. Let's show that $\bar{f}$ has finite fibers of cardinality at most 2 and that the generic fiber has cardinality 2. The fiber of $\bar{f}$ over $\left|I_{F_{5}^{3}}(2)\right| \in\left|I_{S}(2)\right|^{\vee}$ consists of the unique singular point $p$ of $N$. Now let $\Lambda \in\left(W \backslash\left\{\left|I_{F_{5}^{3}}(2)\right|\right\}\right)$, i.e.

$$
\Lambda=f([Z]), \quad[Z] \notin P .
$$

Then $\bar{f}^{-1}(\Lambda)=f^{-1}(\Lambda)$. Let $\Lambda_{0}:=\Lambda \cap\left|I_{F_{5}^{3}}(2)\right|$ and choose $\lambda_{0} \in\left(\Lambda \backslash\left|I_{F_{5}^{3}}(2)\right|\right)$. One has

$$
\bigcap_{\lambda \in \Lambda_{0}} Q_{\lambda}=F_{5}^{3} \cup A_{\Lambda}
$$

where $A_{\Lambda}$ is a plane such that $A_{\Lambda} \cap F_{5}^{3}=C_{\Lambda}$ is a conic. (See the proof of Lemma (4.20) of [12].) We claim that $Q_{\lambda_{0}} \not \supset A_{\Lambda}$ : in fact if $Q_{\lambda_{0}} \supset A_{\Lambda}$ then $C_{\Lambda} \subset S$ contradicting (3.1.4). Thus $Q_{\lambda_{0}} \cap A_{\Lambda}$ is a conic $C_{\Lambda}^{\prime}$. By (3.2.11) the line $\operatorname{span}(Z)$ is contained in $C_{\Lambda}^{\prime}$. Thus $C_{\Lambda}^{\prime}$ is degenerate and $f^{-1}(\Lambda)$ consists of the set of lines contained in $C_{\Lambda}^{\prime}$. This shows that $f^{-1}(\Lambda)=\bar{f}^{-1}(\Lambda)$ has cardinality at most 2. It also follows easily that the generic fiber of $\bar{f}$ consists of 2 distinct points. Since $N$ is normal there is a regular covering involution $\bar{\phi}$ such that $\bar{f}$ factors through the quotient map $N \rightarrow N /\langle\bar{\phi}\rangle$. Since $\bar{f}$ has finite fibers so does $\epsilon$, since the generic fiber of $\bar{f}$ consists of 2 points the map $\epsilon$ is birational. The line-bundle $\bar{f}^{*} \mathcal{O}_{W}(1)$ is ample because $\bar{f}: N \rightarrow W$ has finite fibers, thus $N$ is projective. We know (see (4.3) of [12]) that

$$
\int_{S^{[2]}} c_{1}\left(f^{*} \mathcal{O}_{W}(1)\right)=12
$$

Since $f: S^{[2]} \rightarrow W$ has generic fiber of cardinality 2 we get that $\operatorname{deg} W=6$. 
We will show that the map $\epsilon$ of Lemma (3.7) is an isomorphism. Since $\left(S^{[2]} \backslash\right.$ $P) \cong(N \backslash\{p\})$ the involution $\bar{\phi}$ defines a birational involution

$$
\phi: S^{[2]} \rightarrow S^{[2]} \text {. }
$$

(This is the birational involution of Proposition (4.21) of [12].) Let

$$
\beta: B l_{P}\left(S^{[2]}\right) \rightarrow S^{[2]}
$$

be the blow-up of $P$ : since $P$ is Lagrangian the symplectic form on $S^{[2]}$ induces an isomorphism $N_{P / S^{[2]}} \cong \Omega_{P}^{1}$ and hence the exceptional divisor of $\beta$ is identified with the incidence variety $\Gamma \subset P \times P^{\vee}$ and the restriction of $\beta$ to the exceptional divisor is identified with the projection $\Gamma \rightarrow P$. We abuse notation and view $\Gamma$ as the exceptional divisor in $B l_{P}\left(S^{[2]}\right)$

Lemma 3.8. The map $\phi$ of (3.2.14) is not regular along $P$. There is a regular involution $\widetilde{\phi}: B l_{P}\left(S^{[2]}\right) \rightarrow B l_{P}\left(S^{[2]}\right)$ which is equal to $\phi$ on $\left(S^{[2]} \backslash P\right) \subset B l_{P}\left(S^{[2]}\right)$. There is an identification $P \cong P^{\vee}$ such that $\left.\widetilde{\phi}\right|_{\Gamma}$ is induced by the involution on $P \times P^{\vee}$ which interchanges the factors.

Proof. The eigenspaces of the isometry $H^{2}(\phi)$ on $H^{2}\left(S^{[2]}\right)$ induced by $\phi$ are given by (see (4.3) of [12])

$$
H^{2}(\phi)_{+}=\mathbb{C} f^{*} \mathcal{O}_{W}(1), \quad H^{2}(\phi)_{-}=f^{*} \mathcal{O}_{W}(1)^{\perp} .
$$

Suppose $\phi$ is regular along $P$ : if $D$ is an ample divisor on $S^{[2]}$ then $c_{1}\left(D+\phi^{*} D\right)$ is an ample $\phi$-invariant class, this contradicts $(3.2 .16)$ because $f^{*} \mathcal{O}_{W}(1)$ is not ample. Let $\psi: S^{[2]} \rightarrow \mathcal{X}$ be the flop of $P$ : thus $\psi$ is the inverse of the blowup $B l_{P}\left(S^{[2]}\right) \rightarrow S^{[2]}$ followed by the morphism $B l_{P}\left(S^{[2]}\right) \rightarrow \mathcal{X}$ which contracts $\Gamma$ along the "other" fibration $\Gamma \rightarrow P^{\vee}$. In particular $\mathcal{X}$ contains $P^{\vee}$. Let $\ell \subset P$ and $\ell^{\vee} \subset P^{\vee}$ be lines. The isometry $H^{2}(\psi)$ identifies $\left(\ell^{\vee}\right)^{\perp}$ with $\ell^{\perp}$. In fact we have contractions $c: S^{[2]} \rightarrow N$ and $c^{\vee}: \mathcal{X} \rightarrow N$ which give identifications $\ell^{\perp}=H^{2}(N)=\left(\ell^{\vee}\right)^{\perp}$. In particular $H^{2}(\psi)$ sends a nef divisor class in $\left(\ell^{\vee}\right)^{\perp}$ to a nef divisor class. On the other hand $H^{2}(\psi)$ maps the half-space $\ell_{>0}^{\vee}$ to the halfspace $\ell_{<0}$. By $(3.2 .16)$ we get that $(\psi \circ \phi)^{*}$ maps an ample divisor to an ample divisor: since $(\psi \circ \phi)$ defines a regular map between the complements of subsets of codimension 2 it follows that $(\psi \circ \phi)$ is regular and hence an isomorphism. It follows also that $\phi$ induces a regular involution $\widetilde{\phi}: B l_{P}\left(S^{[2]}\right) \rightarrow B l_{P}\left(S^{[2]}\right)$. Let's show that the restriction of $\widetilde{\phi}$ to $\Gamma$ is as stated. Any automorphism of $\Gamma$ sends 
the projection $\Gamma \rightarrow P$ to itself composed with an automorphism of $P$ or to the projection $\Gamma \rightarrow P^{\vee}$ composed with an automorphism of $P^{\vee}$. Since $\phi$ is not regular the latter holds and it follows that $\left.\widetilde{\phi}\right|_{\Gamma}$ is as stated.

Corollary 3.9. The map $\epsilon: N /\langle\bar{\phi}\rangle \rightarrow W$ of Lemma (3.7) is an isomorphism.

Proof. The quotient $B l_{P}\left(S^{[2]}\right) /\langle\widetilde{\phi}\rangle$ is a projective birational model of $N /\langle\bar{\phi}\rangle$ and hence it is birational to $W$ by Lemma (3.7). The Kodaira dimension of $B l_{P}\left(S^{[2]}\right) /\langle\widetilde{\phi}\rangle$ is 0 hence also the Kodaira dimension of $W$ is 0. By Lemma (3.7) we know that $\operatorname{deg} W=6$ and hence by adjunction $W$ is smooth in codimension 1. Thus $W$ is normal: since $\epsilon$ is birational with finite fibers we get that $\epsilon$ is an isomorphism.

Now we reintroduce the subscript $t$; thus we have $S_{t}, f_{t}, N_{t}, W_{t}$ etc.

Proposition 3.10. Keep notation as above. Let $A \in \Delta^{0}(V)$ and let $t \in T^{\prime \prime \prime}$ such that $X_{A} \cong M_{t}$ - such a $t$ exists by Definition (3.3). Notice that by Proposition (3.1) and Corollary (3.6) we have $Y_{A^{\perp}} \subset\left|I_{S_{t}}(2)\right|^{\vee}$. The following equality holds:

$$
Y_{A^{\perp}}=W_{t}
$$

Proof. Proposition (5.20) of [12] gives that the reduced scheme $\left(Y_{A^{\perp}}\right)_{\text {red }}$ is equal to $W_{t}$ (the hypothesis of that Proposition is that (3.0.2) holds, however the same proof goes through because all that is needed is the validity of (3.0.5)). Now $Y_{A^{\perp}}$ is a degree-6 hypersurface because $Y_{A^{\perp}} \neq \mathbb{P}\left(V^{\vee}\right)$ and on the other hand $W_{t}$ is a degree-6 hypersurface by Lemma (3.7) and hence from $\left(Y_{A^{\perp}}\right)_{r e d}=W_{t}$ we get that $Y_{A^{\perp}}$ is reduced and equal to $W_{t}$.

Let $A \in \Delta^{0}(V)$ and let $t \in T^{\prime \prime \prime}$ such that $X_{A} \cong M_{t}$. Then $\left|I_{F_{5}^{3}}(2)\right| \in W_{t}$ see (3.2.8) - and hence by the above proposition $\left|I_{F_{5}^{3}}(2)\right| \in Y_{A^{\perp}}$; we denote this point by $q_{A^{\perp}}$.

Proposition 3.11. Let $A \in \Delta^{0}(V)$ and let $t \in T^{\prime \prime \prime}$ such that $X_{A} \cong M_{t}$. Then

$$
\text { mult }_{q_{A^{\perp}}} Y_{A^{\perp}}=3 .
$$

Let $y \in\left(Y_{A^{\perp}} \backslash\left\{q_{A^{\perp}}\right\}\right)$ and hence ${\overline{f_{t}}}^{-1}(y)=f_{t}^{-1}(y)$ consists of two points or of one point. 
(a) If $f_{t}^{-1}(y)$ consists of two points then $Y_{A^{\perp}}$ is smooth at $y$.

(b) If $f_{t}^{-1}(y)$ consists of a single point then the analytic germ $\left(Y_{A^{\perp}}, y\right)$ is isomorphic to the product of a smooth 2-dimensional germ and the germ of an $A_{1}$-singularity.

Proof. By Proposition (6.2) of [13] $Y_{A}^{\vee}$ has multiplicity 3 in $\left|I_{F_{5}^{3}}(2)\right|$; this proves (3.2.18). In order to prove (a)-(b) we notice that $Y_{A^{\perp}}=W_{t}$ and by Proposition (3.9) the map $\bar{f}_{t}: N_{t} \rightarrow W_{t}$ is identified with the quotient map $N_{t} \rightarrow N_{t} /\langle\bar{\phi}\rangle$. Since $p_{t}=\bar{f}^{-1}\left(q_{A}\right)$ is the unique singular point of $N_{t}$ this gives Item (a). In order to prove Item (b) we notice that $\left(N_{t} \backslash\left\{p_{t}\right\}\right)=\left(S_{t}^{[2]} \backslash P_{t}\right)$ and that the quotient map $\left(N_{t} \backslash\left\{p_{t}\right\}\right) \rightarrow\left(N_{t} \backslash\left\{p_{t}\right\}\right) /\langle\bar{\phi}\rangle$ is identified with the quotient map $\left(S_{t}^{[2]} \backslash P_{t}\right) \rightarrow\left(S_{t}^{[2]} \backslash P_{t}\right) /\langle\phi\rangle$. By Proposition (4.21) of [12] the restriction of $\phi$ to $\left(S_{t}^{[2]} \backslash P_{t}\right)$ is an anti-symplectic involution and hence its fixed point set is a Lagrangian surface; this proves (b).

Let $A \in \Delta^{0}(V)$; then by (3.2.18) the sextic $Y_{A^{\perp}}$ has a point of multiplicity 3 and hence $A^{\perp} \notin \mathbb{L} \mathbb{G}\left(\wedge^{3} V^{\vee}\right)^{0}$ because if $B \in \mathbb{L} \mathbb{G}\left(\wedge^{3} V^{\vee}\right)^{0}$ then $Y_{B}$ has points of multiplicity at most 2 . Thus

$$
\Delta^{0}(V) \cap \mathbb{L} \mathbb{G}\left(\wedge^{3} V\right)^{00}=\emptyset .
$$

Let $\Delta_{*}^{0}(V) \subset \Delta^{0}(V)$ be the set of points which are smooth points of the projective variety $\left(\mathbb{L} \mathbb{G}\left(\wedge^{3} V\right) \backslash \mathbb{L} \mathbb{G}\left(\wedge^{3} V\right)^{00}\right)$. By Proposition (3.4) we know that $\Delta^{0}(V)$ is locally (in the classical topology) a codimension 1 submanifold of $\mathbb{L} \mathbb{G}\left(\wedge^{3} V\right)$; since $\Delta^{0}(V) \cap \mathbb{L} \mathbb{G}\left(\wedge^{3} V\right)^{00}=\emptyset$ it follows that $\Delta_{*}^{0}(V)$ is open dense in $\Delta^{0}(V)$. We let $T_{*} \subset T^{\prime \prime \prime}$ be the set of $t$ such that $M_{t} \cong X_{A}$ for some $A \in \Delta_{*}^{0}(V)$; since $\Delta_{*}^{0}(V)$ is open dense in $\Delta^{0}(V)$ also $T_{*}$ is open dense in $T^{\prime \prime \prime}$. Let

$$
\mathbb{L} \mathbb{G}\left(\wedge^{3} V\right)_{*}^{0}:=\mathbb{L} \mathbb{G}\left(\wedge^{3} V\right)^{00} \cup \Delta_{*}^{0}(V) .
$$

Proposition 3.12. Keep notation as above. Then $\mathbb{L} \mathbb{G}\left(\wedge^{3} V\right)_{*}^{0}$ is open in $\mathbb{L} \mathbb{G}\left(\wedge^{3} V\right)$ (for the classical topology) and $\Delta_{*}^{0}(V)$ is a non-empty codimension-1 submanifold of $\mathbb{L} \mathbb{G}\left(\wedge^{3} V\right)_{*}^{0}$.

Proof. Let $A \in \Delta_{*}^{0}(V)$. By definition of $\Delta_{*}^{0}(V)$ there exists an open $U \subset$ $\mathbb{L} \mathbb{G}\left(\wedge^{3} V\right)$ such that $U \cap \Delta_{*}^{0}(V)=U \cap\left(\mathbb{L} \mathbb{G}\left(\wedge^{3} V\right) \backslash \mathbb{L} \mathbb{G}\left(\wedge^{3} V\right)^{00}\right)$ and hence $U \subset$ $\mathbb{L} \mathbb{G}\left(\wedge^{3} V\right)_{*}^{0}$; this proves that $\mathbb{L} \mathbb{G}\left(\wedge^{3} V\right)_{*}^{0}$ is open. We have already proved that 
$\Delta_{*}^{0}(V)$ is a non-empty locally closed codimension-1 submanifold of $\mathbb{L} \mathbb{G}\left(\wedge^{3} V\right)$; this gives the second statement of the proposition.

We let

$$
\begin{aligned}
\Delta_{*}^{\infty}\left(V^{\vee}\right) & :=\delta\left(\Delta_{*}^{0}(V)\right), \\
\mathbb{L} \mathbb{G}\left(\wedge^{3} V^{\vee}\right)_{*}^{\infty} & :=\delta\left(\mathbb{L} \mathbb{G}\left(\wedge^{3} V\right)_{*}^{0}\right) .
\end{aligned}
$$

Of course every definition above has a "dual "definition obtained by substituting $V^{\vee}$ to $V$; thus we have $\Delta_{*}^{0}\left(V^{\vee}\right) \subset \mathbb{L} \mathbb{G}\left(\wedge^{3} V^{\vee}\right)^{0}, \Delta_{*}^{\infty}(V) \subset \mathbb{L} \mathbb{G}\left(\wedge^{3} V\right)$ etc. Let

$$
\begin{aligned}
\mathbb{L} \mathbb{G}\left(\wedge^{3} V^{\vee}\right)^{\sharp}:=\mathbb{L} \mathbb{G}\left(\wedge^{3} V^{\vee}\right)_{*}^{0} \cup \mathbb{L} \mathbb{G}\left(\wedge^{3} V^{\vee}\right)_{*}^{\infty}= \\
\quad=\mathbb{L} \mathbb{G}\left(\wedge^{3} V^{\vee}\right)^{00} \cup \Delta_{*}^{0}\left(V^{\vee}\right) \cup \Delta_{*}^{\infty}\left(V^{\vee}\right) .
\end{aligned}
$$

Let $\mathcal{Y}\left(V^{\vee}\right) \subset \mathbb{L} \mathbb{G}\left(\wedge^{3} V^{\vee}\right)^{\sharp} \times \mathbb{P}\left(V^{\vee}\right)$ be the tautological EPW-sextic; thus $\mathcal{Y}\left(V^{\vee}\right) \cap$ $\{B\} \times \mathbb{P}\left(V^{\vee}\right)=Y_{B}$. If $B \in \Delta_{*}^{\infty}\left(V^{\vee}\right)$ then by Proposition (3.11) there is a unique point $q_{B} \in Y_{B}$ of multiplicity strictly greater than 2 ; let

$$
\begin{aligned}
\mathcal{Q}\left(V^{\vee}\right) & :=\left\{\left(B, q_{B}\right) \mid B \in \Delta_{*}^{\infty}\left(V^{\vee}\right)\right\}, \\
\mathcal{Y}\left(V^{\vee}\right)^{\sharp} & :=\mathcal{Y}\left(V^{\vee}\right) \backslash \mathcal{Q}\left(V^{\vee}\right) .
\end{aligned}
$$

Proposition 3.13. There exists a double cover $f: \mathcal{X}\left(V^{\vee}\right) \rightarrow \mathcal{Y}\left(V^{\vee}\right)^{\sharp}$ with the following properties.

(1) Let $\pi: \mathcal{X}\left(V^{\vee}\right) \rightarrow \mathbb{L} \mathbb{G}\left(\wedge^{3} V^{\vee}\right)^{\sharp}$ be the composition of $f$ and the projection $\mathcal{Y}\left(V^{\vee}\right)^{\sharp} \rightarrow \mathbb{L} \mathbb{G}\left(\wedge^{3} V^{\vee}\right)^{\sharp}$; then $\pi$ is a submersion of smooth manifolds.

(2) Let $B \in \mathbb{L} \mathbb{G}\left(\wedge^{3} V^{\vee}\right)_{*}^{0}$; then $\pi^{-1}(B) \cong X_{B}$ and the map $\pi^{-1}(B) \rightarrow Y_{B}$ defined by $f$ is isomorphic to the natural double cover $X_{B} \rightarrow Y_{B}$.

(3) Let $B \in \Delta_{*}^{\infty}\left(V^{\vee}\right)$ and $A:=B^{\perp}$. Since $A \in \Delta_{*}^{0}(V)$ there exists $t \in T_{*}$ such that $X_{A} \cong M_{t}$ and $Y_{A} \cong \Sigma_{t}^{\prime}$. Then $\pi^{-1}(B) \cong\left(S_{t}^{[2]} \backslash\left\{P_{t}\right\}\right)$ and the map $\pi^{-1}(B) \rightarrow\left(Y_{B} \backslash\left\{q_{B}\right\}\right)$ defined by $f$ is isomorphic to the double cover $\left(S_{t}^{[2]} \backslash\left\{P_{t}\right\}\right) \rightarrow\left(W_{t} \backslash\left|I_{F_{5}^{3}}(2)\right|\right)$ given by the restriction of (3.2.7). (Recall that $\left(Y_{B} \backslash\left\{q_{B}\right\}\right) \cong\left(W_{t} \backslash\left|I_{F_{5}^{3}}(2)\right|\right)$ by Proposition (3.10)).

Proof. Let $B \in \mathbb{L} \mathbb{G}\left(\wedge^{3} V^{\vee}\right)^{\sharp}$. Then $Y_{B} \neq \mathbb{P}\left(V^{\vee}\right)$ by Corollary (3.6); thus the map $\lambda_{B}$ defined in Section (1) (with $V^{\vee}$ replacing $V$ ) is non-zero and $Y_{B}$ is the zeroscheme of $\operatorname{det}\left(\lambda_{B}\right)$. Since $Y_{B}$ is a Lagrangian degeneracy locus there exists locally 
in $\mathbb{P}\left(V^{\vee}\right)$ a symmetric map of vector-bundles giving a resolution of $\operatorname{coker}\left(\lambda_{B}\right)$, i.e. we can cover $\mathbb{P}\left(V^{\vee}\right)$ by open sets $U$ such that on each $U$ we have a locally-free resolution

$$
\left.0 \rightarrow E_{U} \stackrel{\alpha_{U}}{\longrightarrow} E_{U}^{\vee} \longrightarrow \operatorname{coker}\left(\lambda_{B}\right)\right|_{U} \rightarrow 0
$$

where $\alpha_{U}$ is a symmetric map of vector-bundles. Furthermore if $F \hookrightarrow \wedge^{3} V^{\vee} \otimes$ $\mathcal{O}_{\mathbb{P}\left(V^{\vee}\right)}$ is the Lagrangian sub-vector-bundle defined in Section (1) (with $V^{\vee}$ replacing $V$ ) then there is an isomorphism $F_{p} \cap B \cong \operatorname{ker}\left(\alpha_{p}\right)$ for all $p \in U$. Thus if $\operatorname{dim}\left(F_{p} \cap B\right) \geq r$ then mult $_{p}\left(Y_{B}\right) \geq r$. Let $p \neq q_{B}$. Then mult $_{p}\left(Y_{B}\right) \leq 2$ by Proposition (3.11) and hence $\operatorname{dim}\left(F_{p} \cap B\right) \leq 2$. Furthermore one of the following holds:

(1) If $\operatorname{dim}\left(F_{p} \cap B\right)=1$ then locally around $p$ we have $\operatorname{coker}\left(\lambda_{B}\right) \cong i_{*} \mathcal{O}_{Y_{B}}$ where $i: Y_{B} \hookrightarrow \mathbb{P}\left(V^{\vee}\right)$ is the inclusion.

(2) If $\operatorname{dim}\left(F_{p} \cap B\right)=2$ there exist an open (in the classical topology) $U \subset$ $\mathbb{P}\left(V^{\vee}\right)$ containing $p$, holomorphic functions $x, y, z$ on $U$ vanishing at $p$ with linearly independent differentials and an exact sequence

$$
\left.0 \rightarrow \mathcal{O}_{U}^{2} \stackrel{M}{\longrightarrow} \mathcal{O}_{U}^{2} \longrightarrow \operatorname{coker}\left(\lambda_{B}\right)\right|_{U} \rightarrow 0
$$

where $M$ is the map defined by the matrix

$$
\left(\begin{array}{l}
x y \\
y z
\end{array}\right) .
$$

In particular we see that there exists a sheaf $\zeta_{B}$ on $\left(Y_{B} \backslash\left\{q_{B}\right\}\right)$ such that outside $q_{B}$ we have coker $\left(\lambda_{B}\right)=i_{*}^{0} \zeta_{B}$ where $i^{0}:\left(Y_{B} \backslash\left\{q_{B}\right\}\right) \hookrightarrow\left(\mathbb{P}\left(V^{\vee}\right) \backslash\left\{q_{B}\right\}\right)$ is the inclusion. From the local description of $\operatorname{coker}\left(\lambda_{B}\right)$ given above we also get a canonical isomorphism of sheaves on $\left(\mathbb{P}\left(V^{\vee}\right) \backslash\left\{q_{B}\right\}\right)$ :

$$
\left.\operatorname{Ext}\left(\operatorname{coker}\left(\lambda_{B}\right), \mathcal{O}_{\mathbb{P}\left(V^{\vee}\right)}\right)\right|_{\mathbb{P}\left(V^{\vee}\right) \backslash\left\{q_{B}\right\}} \cong i_{*}^{0}\left(\zeta_{B}^{\vee} \otimes N_{Y_{B} / \mathbb{P}\left(V^{\vee}\right)}\right) .
$$

(See Proposition (4.3) of [13].) Let $B^{\vee} \subset \wedge^{3} V^{\vee}$ be a Lagrangian subspace complementary to $B$; thus we have a direct sum decomposition

$$
\wedge^{3} V=B \oplus B^{\vee}
$$

Then $\lambda_{B}$ can be identified with the map of vector-bundles $F \rightarrow B^{\vee} \otimes \mathcal{O}_{\mathbb{P}\left(V^{\vee}\right)}$ associated to Decomposition (3.2.31). Let $\mu_{B}: F \rightarrow B \otimes \mathcal{O}_{\mathbb{P}\left(V^{\vee}\right)}$ be the "other" map 
associated to Decomposition (3.2.31). The diagram

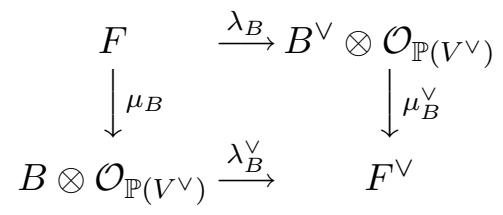

is commutative because $F \stackrel{\left(\mu_{B}, \lambda_{B}\right)}{\longrightarrow}\left(B \oplus B^{\vee}\right) \otimes \mathcal{O}_{\mathbb{P}\left(V^{\vee}\right)}$ is a Lagrangian embedding. The map $\lambda_{B}$ is an injection of sheaves because $Y_{B} \neq \mathbb{P}\left(V^{\vee}\right)$ and hence also $\lambda_{B}^{\vee}$ is an injection of sheaves. Thus there is a unique $\beta_{B}: \operatorname{coker}\left(\lambda_{B}\right) \longrightarrow$ $\operatorname{Ext}^{1}\left(\operatorname{coker}\left(\lambda_{B}\right), \mathcal{O}_{\mathbb{P}\left(V^{\vee}\right)}\right)$ making the following diagram commutative with exact horizontal sequences:

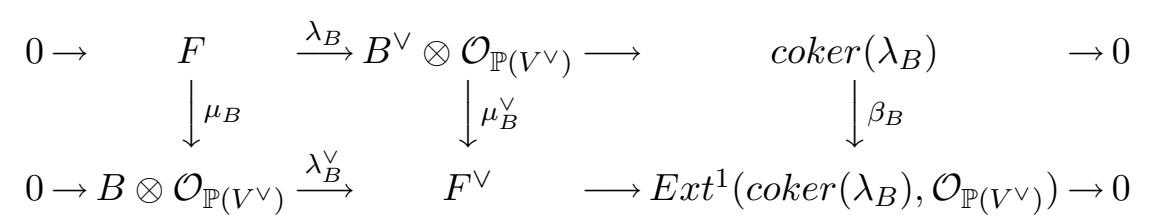

By Isomorphism (3.2.30) we get that the restriction of $\beta_{B}$ to $\left(\mathbb{P}\left(V^{\vee}\right) \backslash\left\{q_{B}\right\}\right)$ defines a map of sheaves on $\left(Y_{B} \backslash\left\{q_{B}\right\}\right)$

$$
\zeta_{B} \rightarrow \zeta_{B}^{\vee}(6)
$$

Since $F \stackrel{\left(\mu_{B}, \lambda_{B}\right)}{\longrightarrow}\left(B \oplus B^{\vee}\right) \otimes \mathcal{O}_{\mathbb{P}(V \vee)}$ is an injection of vector-bundles the above map is an isomorphism - this follows from Claim (4.5) of [13]. Let $\xi_{B}:=\zeta_{B}(-3)$; then (3.2.34) defines an isomorphism $\xi_{B} \stackrel{\sim}{\longrightarrow} \xi_{B}^{\bigvee}$ which is symmetric and hence it

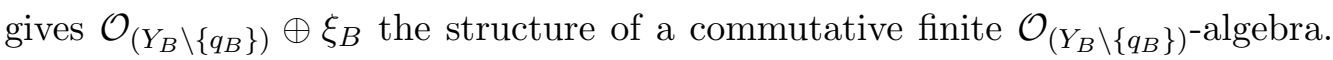
Let $X_{B}:=\operatorname{Spec}\left(\mathcal{O}_{\left(Y_{B} \backslash\left\{q_{B}\right\}\right)} \oplus \xi_{B}\right)$ and $f_{B}: X_{B} \rightarrow\left(Y_{B} \backslash\left\{q_{B}\right\}\right)$ be the structure map: clearly $f_{B}$ is finite of degree 2 . The above construction is the analogue of the construction of the natural double cover $X_{B} \rightarrow Y_{B}$ for $B \in \mathbb{L} \mathbb{G}\left(\wedge^{3} V^{\vee}\right)^{0}$; thus we have a double cover $f: \mathcal{X}\left(V^{\vee}\right) \rightarrow \mathcal{Y}^{\sharp}\left(V^{\vee}\right)$ such that Item (2) holds and such that for $B \in \Delta_{*}^{\infty}\left(V^{\vee}\right)$ we have $\pi^{-1}(B)=X_{B}$ and the map $\pi^{-1}(B) \rightarrow\left(Y_{B} \backslash\left\{q_{B}\right\}\right)$ is the structure map $f_{B}$ defined above. It remains to prove that Items (1) and (3) hold. Let

$$
\begin{aligned}
& C_{B}:=\left\{p \in\left(\operatorname{sing} Y_{B} \backslash q_{B}\right) \mid \operatorname{dim}\left(F_{p} \cap B\right)=1\right\} \\
& D_{B}:=\left\{p \in\left(\operatorname{sing} Y_{B} \backslash q_{B}\right) \mid \operatorname{dim}\left(F_{p} \cap B\right)=2\right\} .
\end{aligned}
$$

Thus $\left(\operatorname{sing} Y_{B} \backslash q_{B}\right)=C_{B} \amalg D_{B}$. The local description given above of $\operatorname{coker}\left(\lambda_{B}\right)$ near $p \in\left(Y_{B} \backslash q_{B}\right)$ shows that both $C_{B}$ and $D_{B}$ are smooth and closed (in 
$\left.\left(Y_{B} \backslash q_{B}\right)\right)$. Let $p \in\left(Y_{B} \backslash q_{B}\right)$; the map $f_{B}$ behaves differently depending on whether $p \in C_{B}$ or $p \in D_{B}$ : in fact (see [13])

( $\alpha$ ) If $p \in C_{B}$ then $f_{B}$ is unramified over $p$.

$(\beta)$ If $p \in D_{B}$ then $f_{B}$ is ramified over $p$ and $X_{B}$ is smooth at $f_{B}^{-1}(p)$.

Let's prove that

$$
X_{B} \cong\left(S_{t}^{[2]} \backslash P_{t}\right)
$$

Let $B^{\prime} \in \mathbb{L} \mathbb{G}\left(\wedge^{3} V^{\vee}\right)_{*}^{0}$ and define $D_{B^{\prime}}$ as above. Then $D_{B^{\prime}}$ is not empty in fact it is a surface; it follows that $D_{B} \neq \emptyset$ and by Item $(\beta)$ above we get that the étale covering

$$
\left(X_{B} \backslash f_{B}^{-1}\left(\operatorname{sing} Y_{B}\right)\right) \longrightarrow\left(Y_{B} \backslash \operatorname{sing} Y_{B}\right)
$$

is not trivial. On the other hand let $t \in T_{*}$ such that $X_{A^{\perp}} \cong M_{t}$ and $Y_{A^{\perp}} \cong \Sigma_{t}^{\prime}$. Let $f_{t}: S_{t}^{[2]} \rightarrow Y_{B}$ be the map defined by (3.2.7); then $f_{t}$ defines an étale double cover

$$
\left(S_{t}^{[2]} \backslash f_{t}^{-1}\left(\operatorname{sing} Y_{B}\right)\right) \longrightarrow\left(Y_{B} \backslash \operatorname{sing} Y_{B}\right) .
$$

Now $S_{t}^{[2]}$ is simply connected and $f_{t}^{-1}\left(\operatorname{sing} Y_{B}\right)$ has codimension 2 in $S_{t}^{[2]}$ hence $\left(S_{t}^{[2]} \backslash f_{t}^{-1}\left(\operatorname{sing} Y_{B}\right)\right)$ is simply connected. Thus $\pi_{1}\left(Y_{B} \backslash \operatorname{sing} Y_{B}\right) \cong \mathbb{Z} /(2)$ and hence there is a unique non-trivial double cover of $\left(Y_{B} \backslash \operatorname{sing} Y_{B}\right)$ and it is given by (3.2.39). Since (3.2.38) is a non-trivial double cover it follows that $C_{B}=\emptyset$ and that (3.2.37) holds. This proves Items (1)-(3) of the proposition.

3.3. Extension of the local period map across $\Delta_{*}^{\infty}\left(V^{\vee}\right)$. We will prove that the local period map extends across $\Delta_{*}^{\infty}\left(V^{\vee}\right)$. Let $\pi^{0}: \mathcal{X}\left(V^{\vee}\right)_{*}^{0} \rightarrow \mathbb{L} \mathbb{G}\left(\wedge^{3} V^{\vee}\right)_{*}^{0}$ be the tautological family of double EPW-sextics i.e. the restriction of the map $\pi$ of Proposition (3.13) to $\pi^{-1}\left(\mathbb{L} \mathbb{G}\left(\wedge^{3} V^{\vee}\right)_{*}^{0}\right)$. Since $\pi^{0}$ is proper it defines the variation of Hodge structures

$$
\left(R^{2} \pi_{*}^{0} \mathbb{Z}, F^{p}\right)
$$

where $F^{0} \supset F^{1} \supset F^{2}$ is the Hodge filtration of $\left(R^{2} \pi_{*}^{0} \mathbb{Z}\right) \otimes \mathcal{O}_{\mathbb{L} \mathbb{G}\left(\wedge^{3} V^{\vee}\right)_{*}^{0}}$ i.e. the fiber of $F^{p}$ over $A$ is

$$
F_{A}^{p}=F^{p} H^{2}\left(X_{A}\right):=\bigoplus_{p^{\prime} \geq p} H^{p^{\prime}, 2-p^{\prime}}\left(X_{A}\right)
$$


Furthermore we have the symmetric section

$$
\mathbb{B} \in H^{0}\left(\left(R^{2} \pi_{*}^{0} \mathbb{Q}\right) \otimes\left(R^{2} \pi_{*}^{0} \mathbb{Q}\right)\right)
$$

which gives the Beauville-Bogomolov bilinear form on $H^{2}\left(X_{A}\right)$ for every $A \in$ $\mathbb{L} \mathbb{G}\left(\wedge^{3} V^{\vee}\right)_{*}^{0}$.

Proposition 3.14. There exist

(1) a local system $\mathbb{H}\left(V^{\vee}\right)$ on $\mathbb{L} \mathbb{G}\left(\wedge^{3} V^{\vee}\right)^{\sharp}$,

(2) a decreasing filtration of $\mathbb{H}\left(V^{\vee}\right) \otimes \mathcal{O}_{\mathbb{L} \mathbb{G}\left(\wedge^{3} V^{\vee}\right)^{\sharp}}$ by holomorphic sub-bundles $\widetilde{F}^{0} \supset \widetilde{F}^{1} \supset \widetilde{F}^{2}$

(3) a symmetric section $\left.\widetilde{\mathbb{B}} \in H^{0}\left(\left(\mathbb{H}\left(V^{\vee}\right) \otimes \mathbb{H}\left(V^{\vee}\right)\right) \otimes \mathbb{Q}\right)\right)$

such that the following hold:

(a) $\left(\mathbb{H}\left(V^{\vee}\right), \widetilde{F}^{p}\right)$ extends the variation of Hodge structures (3.3.1).

(b) Let $B \in \Delta_{*}^{\infty}\left(V^{\vee}\right)$ and $t \in T_{*}$ such that $M_{t} \cong X_{B^{\perp}}$; then there exists an isomorphism of Hodge structures

$$
\left(\mathbb{H}\left(V^{\vee}\right)_{B}, \widetilde{F}_{B}^{p}\right) \cong H^{2}\left(S_{t}^{[2]}\right) .
$$

(c) The restriction of $\widetilde{\mathbb{B}}$ to $\mathbb{L} \mathbb{G}\left(\wedge^{3} V^{\vee}\right)_{*}^{0}$ is equal to $\mathbb{B}$.

(d) Let $B \in \Delta_{*}^{\infty}\left(V^{\vee}\right)$ and $t \in T_{*}$ such that $M_{t} \cong X_{B^{\perp}}$; then Isomorphism (3.3.4) is an isometry between $\left(\mathbb{H}\left(V^{\vee}\right)_{B}, \widetilde{\mathbb{B}}_{B}\right)$ and $H^{2}\left(S_{t}^{[2]}\right)$ equipped with the Beauville-Bogomolov symmetric bilinear form.

Proof. Let $\mathcal{U} \subset \mathbb{L} \mathbb{G}\left(\wedge^{3} V^{\vee}\right)^{\sharp}$ be an open ball. We assume that $\mathcal{U}$ is small: then there exists a hyperplane $H \subset \mathbb{P}\left(V^{\vee}\right)$ such that $q_{B} \notin H$ for all $B \in \mathcal{U} \cap \Delta_{*}^{\infty}\left(V^{\vee}\right)$. Let $\pi$ and $f$ be as in Proposition (3.13); we let

$$
\begin{aligned}
\mathcal{X}\left(V^{\vee}\right) \mathcal{U} & :=\pi^{-1}(\mathcal{U}), \\
Z(\mathcal{U}, H) & :=\mathcal{X}\left(V^{\vee}\right) \mathcal{U} \cap f^{-1} H .
\end{aligned}
$$

Let $\rho: Z(\mathcal{U}, H) \rightarrow \mathcal{U}$ be given by the restriction of $f$. By our choice of $H$ the map $\rho$ is proper submersive with fibers smooth 3 -folds. Thus we have a variation of Hodge structures $\left(R^{2} \rho_{*} \mathbb{Z}, F^{p}\right)$; we denote it by $\left(\mathbb{H}(\mathcal{U}, H), F^{p}(\mathcal{U}, H)\right)$. Let $B \in\left(\mathcal{U} \backslash \Delta_{*}^{\infty}\right)$; then $r h o^{-1}(B)$ is an ample divisor on $X_{B}$ and hence we have a canonical isomorphism

$$
\left.\left.\left(\mathbb{H}(\mathcal{U}, H), F^{p}(\mathcal{U}, H)\right)\right|_{\left(\mathcal{U} \backslash \Delta_{*}^{\infty}\right)} \cong\left(R^{2} \rho_{*} \mathbb{Z}, F^{p}\right)\right|_{\left(\mathcal{U} \backslash \Delta_{*}^{\infty}\right)} .
$$


This shows that $\left(\mathbb{H}(\mathcal{U}, H), F^{p}(\mathcal{U}, H)\right)$ does not depend on the choice of $H$ and that the collection of $\left(\mathbb{H}(\mathcal{U}, H), F^{p}(\mathcal{U}, H)\right)$ gives an extension $\left(\mathbb{H}\left(V^{\vee}\right), \widetilde{F}^{p}\right)$ of the variation of Hodge structures (3.3.1). Now let's prove Item (b). Let $B \in(\mathcal{U} \cap$ $\left.\Delta_{*}^{\infty}\left(V^{\vee}\right)\right)$ and let $t$ be as in Item (b). Let $f_{t}: S_{t}^{[2]} \rightarrow W_{t}=Y_{B}$ be the map given by (3.2.7). Then $\rho^{-1}(B)=f_{t}^{-1} H$ and since $f_{t}$ is semi-small the restriction map $H^{2}\left(S_{t}^{[2]}\right) \rightarrow H^{2}\left(f_{t}^{-1} H\right)$ is an isomorphism of (integral) Hodge structures; this proves Item (b) because $\left(\mathbb{H}\left(V^{\vee}\right)_{B}, \widetilde{F}^{p}\right)$ is isomorphic to the Hodge structure on $H^{2}\left(f_{t}^{-1} H\right)$ by definition. We define $\widetilde{\mathbb{B}}$ as follows. Let $\mathcal{U}$ and $\rho: Z(\mathcal{U}, H) \rightarrow \mathcal{U}$ be as above; let $Z_{B}:=\rho^{-1}(B)$. For $B \in \mathcal{U}$ we have the Lefschetz decomposition

$$
\mathbb{H}\left(V^{\vee}\right)_{B}=H^{2}\left(Z_{B} ; \mathbb{Q}\right)=\left.\mathbb{Q} c_{1}\left(L_{B}\right)\right|_{Z_{B}} \oplus H^{2}\left(Z_{B} ; \mathbb{Q}\right)_{\text {prim }}
$$

where $L_{B}$ is the tautological ample line-bundle on $X_{B}$. We let $\widetilde{\mathbb{B}}(\mathcal{U}, H)_{B}$ be the symmetric bilinear form on $\mathbb{H}\left(V^{\vee}\right)_{B}$ characterized by the following requirements:

$(\alpha)$ Decomposition (3.3.8) is orthogonal for $\widetilde{\mathbb{B}}(\mathcal{U}, H)_{B}$.

$(\beta) \widetilde{\mathbb{B}}_{B}\left(\left.c_{1}\left(L_{B}\right)\right|_{Z_{B}},\left.c_{1}\left(L_{B}\right)\right|_{Z_{B}}\right)=2$.

$(\gamma)$ If $x, y \in H^{2}\left(Z_{B} ; \mathbb{Q}\right)_{\text {prim }}$ then $\widetilde{\mathbb{B}}(\mathcal{U}, H)_{B}(x, y)=\frac{1}{2} \int_{Z_{B}} c_{1}\left(L_{B}\right) \wedge x \wedge y$.

Since the Lefscethz decomposition is flat for the Gauss-Manin connection we have a well-defined section $\widetilde{\mathbb{B}}(\mathcal{U}, H) \in H^{0}\left(\mathbb{H}\left(V^{\vee}\right) \mid \mathcal{U}\right)$ with value $\widetilde{\mathbb{B}}(\mathcal{U}, H)_{B}$ at $B \in \mathcal{U}$. Let $B \in\left(\mathcal{U} \backslash \Delta_{*}^{\infty}\left(V^{\vee}\right)\right.$ and let $\iota: Z_{B} \hookrightarrow X_{B}$ be the inclusion. If $\xi_{1}, \xi_{2}, \xi_{3} \in H^{2}\left(X_{B}\right)$ then

$\int_{Z_{B}} \iota^{*} \xi_{1} \wedge \iota^{*} \xi_{2} \wedge \iota^{*} \xi_{3}=\int_{X_{B}} c_{1}\left(L_{B}\right) \wedge \xi_{1} \wedge \xi_{2} \wedge \xi_{3}=\frac{1}{2} \sum_{\sigma \in \mathcal{S}_{3}}\left(c_{1}\left(L_{B}\right), \xi_{\sigma(1)}\right) \cdot\left(\xi_{\sigma(2)}, \xi_{\sigma(3)}\right)$.

It follows from this that $\widetilde{\mathbb{B}}(\mathcal{U}, H)$ does not depend on $H$ and that the collection of $\widetilde{\mathbb{B}}(\mathcal{U}, H)$ 's defines an extension of $\mathbb{B}$. Item (d) holds because Formula (3.3.9) holds if we replace $X_{B}$ by $S_{t}^{[2]}$.

The map $\delta$ defines an isomorphism

$$
\begin{gathered}
\mathbb{L} \mathbb{G}\left(\wedge^{3} V\right)^{\sharp} \stackrel{\sim}{\longrightarrow} \mathbb{L} \mathbb{G}\left(\wedge^{3} V\right)^{\sharp} \\
A \quad \mapsto \delta(A)=A^{\perp} .
\end{gathered}
$$

From now on we will denote by $\delta$ what is actually the restriction of $\delta$ to $\mathbb{L} \mathbb{G}\left(\wedge^{3} V\right)^{\sharp}$. Let $\mathcal{U} \subset \mathbb{L} \mathbb{G}\left(\wedge^{3} V^{\vee}\right)^{\sharp}$ be an open set: then we have two local systems, namely

$$
\left.\mathbb{H}(V)\right|_{\mathcal{U}},\left.\quad \mathbb{H}\left(V^{\vee}\right)\right|_{\delta(\mathcal{U})}
$$


Definition 3.15. Assume that both local systems (3.3.11) are trivial. A marking of $\left.\mathbb{H}(V)\right|_{\mathcal{U}}$ is an isomorphism $\Psi:\left.\mathbb{H}(V)\right|_{\mathcal{U}} \stackrel{\sim}{\longrightarrow} \mathcal{U} \times \widetilde{\Lambda}$ such that:

(1) $\widetilde{\mathbb{B}}_{A}(x, y)=(\Psi(x), \Psi(y))$ for every $A \in \mathcal{U}$ and $x, y \in \mathbb{H}(V)_{A}$, and

(2) $\Psi$ sends the flat section $\left.A \mapsto c_{1}\left(L_{A}\right)\right|_{Z(\mathcal{U}, H)}$ (notation as in the proof of Proposition (3.14)) to $\mathcal{U} \times u$ where $u$ is given by (1.0.8).

We define similarly a marking $\Psi$ of $\left.\mathbb{H}\left(V^{\vee}\right)\right|_{\delta(\mathcal{U})}$.

Of course if $\mathcal{U}$ is a small open ball then both local systems (3.3.11) are trivial. Let $\Phi$ and $\Psi$ be markings of $\left.\mathbb{H}(V)\right|_{\mathcal{U}}$ and $\left.\mathbb{H}\left(V^{\vee}\right)\right|_{\delta(\mathcal{U})}$ respectively. Then we have holomorphic local period maps

$$
\begin{array}{lcc}
\mathcal{U} \stackrel{\mathcal{P}_{\Psi}}{\longrightarrow} \mathcal{D}_{2} & \delta(\mathcal{U}) \stackrel{\mathcal{P}_{\Phi}}{\longrightarrow} & \mathcal{D}_{2} \\
A \mapsto \Phi_{\mathbb{C}}\left(F_{A}^{2}\right) & B \mapsto \Phi_{\mathbb{C}}\left(F_{B}^{2}\right) .
\end{array}
$$

The restrictions of $\mathcal{P}_{\Psi}$ and $\mathcal{P}_{\Phi}$ to $\mathcal{U} \backslash \Delta_{*}^{\infty}(V)$ and $\delta(\mathcal{U}) \backslash \Delta_{*}^{\infty}\left(V^{\vee}\right)$ respectively are local period maps for the families of double EPW-sextics parametrized by $\mathbb{L} \mathbb{G}\left(\wedge^{3} V\right)^{0}$ and $\mathbb{L} \mathbb{G}\left(\wedge^{3} V^{\vee}\right)^{0}$ respectively. We will be interested in comparing $\mathcal{P}_{\Psi}(A)$ and $\mathcal{P}_{\Phi}\left(A^{\perp}\right)$ for $A \in \Delta_{*}^{0}(V)$ - thus $A^{\perp} \notin \mathbb{L} \mathbb{G}\left(\wedge^{3} V^{\vee}\right)^{0}$. Let $t \in T_{*}$ be such that $M_{t} \cong X_{A}$. By (3.3.4) the marking $\Phi$ defines a marking $\Phi_{A^{\perp}}$ of $\left(S_{t}^{[2]}, f_{t}^{*} \mathcal{O}_{Y_{B}}(1)\right)$ and

$$
\mathcal{P}_{\Phi}\left(A^{\perp}\right)=\mathcal{P}_{\Phi_{A^{\perp}}}\left(S_{t}^{[2]}, f_{t}^{*} \mathcal{O}_{Y_{B}}(1)\right)
$$

\section{Proof of Theorem (1.1)}

If $A \in \mathbb{L} \mathbb{G}\left(\wedge^{3} V\right)^{00}$ we have smooth double covers $f_{A}: X_{A} \rightarrow Y_{A}$ and $f_{A^{\perp}}: X_{A^{\perp}} \rightarrow$ $Y_{A^{\perp}}$ : we will show that $X_{A}$ and $X_{A^{\perp}}$ are "isogenous". Given a small open $\mathcal{U} \subset \mathbb{L} \mathbb{G}\left(\wedge^{3} V\right)^{\sharp}$ we may consider markings $\Psi$ and $\Phi$ of $\left.\mathbb{H}(V)\right|_{\mathcal{U}}$ and $\left.\mathbb{H}\left(V^{\vee}\right)\right|_{\delta(\mathcal{U})}$ respectively and the associated local period maps $\mathcal{P}_{\Psi}$ and $\mathcal{P}_{\Phi}$. We will show that locally near $\Delta_{*}^{0}(V)$ we may choose $\Psi$ and $\Phi$ so that $\mathcal{P}_{\Phi} \circ \delta$ is either $r \circ \mathcal{P}$ or the composition of $r \circ \mathcal{P}$ with a certain specific reflection. In the final subsection we will rule out the latter case by considering the monodromy action; by analytic continuation this will prove Theorem (1.1). 
4.1. Isogeny between $X_{A}$ and $X_{A^{\perp}}$. Let $A \in \mathbb{L} \mathbb{G}\left(\wedge^{3} V\right)^{00}$ and set $L_{A}:=$ $f_{A}^{*} \mathcal{O}_{Y_{A}}(1), L_{A^{\perp}}:=f_{A^{\perp}}^{*} \mathcal{O}_{Y_{A^{\perp}}}(1)$. Let

$$
\begin{aligned}
H^{2}\left(X_{A}\right)_{\text {prim }} & :=c_{1}\left(L_{A}\right)^{\perp} \subset H^{2}\left(X_{A}\right), \\
H^{2}\left(X_{A^{\perp}}\right)_{\text {prim }} & :=c_{1}\left(L_{A^{\perp}}\right)^{\perp} \subset H^{2}\left(X_{A^{\perp}}\right) .
\end{aligned}
$$

Proposition 4.1. Let $A \in \mathbb{L} \mathbb{G}\left(\wedge^{3} V\right)^{00}$. There exists an isomorphism of rational Hodge structures

$$
g_{A}: H^{2}\left(X_{A}\right)_{\text {prim }} \stackrel{\sim}{\longrightarrow} H^{2}\left(X_{A^{\perp}}\right)_{\text {prim }}
$$

well-defined up to \pm 1 and such that for $\gamma \in H^{2}\left(X_{A}\right)_{\text {prim }}$

$$
(\gamma, \gamma)_{X_{A}}=\left(g_{A}(\gamma), g_{A}(\gamma)\right)_{X_{A^{\perp}}}
$$

Proof. We recall that $Y_{A^{\perp}}=Y_{A}^{\vee}$, see Corollary (3.6). Let $\Gamma_{A} \subset Y_{A} \times Y_{A^{\perp}}$ be the closure of the Gauss maps:

$$
\Gamma_{A}:=\overline{\left\{\left(p, T_{p} Y_{A}\right) \mid p \in Y_{A}^{s m}\right\}}=\overline{\left\{\left(T_{q} Y_{A^{\perp}}, q\right) \mid q \in Y_{A^{\perp}}^{s m}\right\}} .
$$

Since $A \in \mathbb{L} \mathbb{G}\left(\wedge^{3} V\right)^{0}$ the germ of $Y_{A}$ at each of its singular points is isomorphic to the product of $\left(\mathbb{C}^{2}, 0\right)$ and an $A_{1}$-singularity (see Proposition (2.8) of [13]) and similarly for $Y_{A^{\perp}}$. Thus the projection $\Gamma_{A} \rightarrow Y_{A}$ is identified with the blow-up $\widetilde{Y}_{A} \rightarrow Y_{A}$ of $\operatorname{sing}\left(Y_{A}\right)$. Similarly the projection $\Gamma_{A} \rightarrow Y_{A^{\perp}}$ is identified with the blow-up $\widetilde{Y}_{A^{\perp}} \rightarrow Y_{A^{\perp}}$ of $\operatorname{sing}\left(Y_{A^{\perp}}\right)$. Thus $\Gamma_{A}$ defines an isomorphism $\widetilde{Y}_{A} \stackrel{\sim}{\longrightarrow} \widetilde{Y}_{A^{\perp}}$ and hence it gives an isomorphism of integral Hodge structures

$$
H^{4}\left(\widetilde{Y}_{A}\right) \stackrel{\sim}{\longrightarrow} H^{4}\left(\widetilde{Y}_{A^{\perp}}\right) .
$$

The cohomology groups $H^{4}\left(\widetilde{Y}_{A}\right)$ and $H^{4}\left(X_{A}\right)$ are related as follows. Let $\phi_{A}: X_{A} \rightarrow$ $X_{A}$ be the involution covering $f_{A}: X_{A} \rightarrow Y_{A}$. Let $F_{A} \subset X_{A}$ be the fixed locus of $\phi_{A}$ : this is a Lagrangian smooth surface in $X_{A}$ because $\phi_{A}$ is anti-symplectic. Let $\widetilde{X}_{A} \rightarrow X_{A}$ be the blow-up of $F_{A}$; we have an isomorphism of integral Hodge structures

$$
H^{4}\left(\widetilde{X}_{A}\right) \cong H^{4}\left(X_{A}\right) \oplus H^{2}\left(F_{A}\right)(-1) .
$$

The involution $\phi_{A}$ lifts to an involution $\widetilde{\phi}_{A}: \widetilde{X}_{A} \rightarrow \widetilde{X}_{A}$ and $\widetilde{Y}_{A} \cong \widetilde{X}_{A} /\left\langle\widetilde{\phi}_{A}\right\rangle$. Thus we have an isomorphism of rational Hodge structures

$$
H^{4}\left(\widetilde{Y}_{A}\right) \cong H^{4}\left(\widetilde{X}_{A}\right)^{\left\langle\tilde{\phi}_{A}\right\rangle} \cong H^{4}\left(X_{A}\right)^{\left\langle\phi_{A}\right\rangle} \oplus H^{2}\left(F_{A}\right)(-1) .
$$


Since $X_{A}$ is a deformation of $(K 3)^{[2]}$ we have an isomorphism of rational Hodge Structures $\operatorname{Sym}^{2} H^{2}\left(X_{A}\right) \stackrel{\sim}{\longrightarrow} H^{4}\left(X_{A}\right)$ defined by cup-product. The action of $\phi_{A}$ on $H^{2}\left(X_{A}\right)$ has $(+1)$-eigenspace generated by $c_{1}\left(L_{A}\right)$ and $(-1)$-eigenspace equal to $H^{2}\left(X_{A}\right)_{\text {prim }}$ thus we get an isomorphism of rational Hodge structures

$$
H^{4}\left(X_{A}\right)^{\left\langle\phi_{A}\right\rangle}=\mathbb{C} c_{1}\left(L_{A}\right)^{2} \oplus \operatorname{Sym}^{2} H^{2}\left(X_{A}\right)_{\text {prim }} .
$$

The right-hand side of the above equality contains a rational $(2,2)$ class $q_{A}^{\vee}$ defined by "inverting"the Beauville-Bogomolov bilinear form (see Section (3) of [14]); let $W_{A}:=H^{2}\left(X_{A}\right)_{\text {prim }} \cap\left(q_{A}^{\vee}\right)^{\perp}$. One has a decomposition of rational Hodge Structures (Claim (3.1) of [14])

$$
\mathbb{C} c_{1}\left(L_{A}\right)^{2} \oplus \mathbb{C} q_{A}^{\vee} \oplus W_{A} .
$$

Of course we have analogous notions for $X_{A^{\perp}}$ and hence (4.1.6) defines an isomorphism of rational H.S.'s

$$
\begin{aligned}
\mathbb{C} c_{1}\left(L_{A}\right)^{2} \oplus \mathbb{C} q_{A}^{\vee} \oplus W_{A} \oplus H^{2}\left(F_{A}\right)(-1) & \cong \\
& \mathbb{C} c_{1}\left(L_{A^{\perp}}\right)^{2} \oplus \mathbb{C} q_{A^{\perp}}^{\vee} \oplus W_{A^{\perp}} \oplus H^{2}\left(F_{A^{\perp}}\right)(-1) .
\end{aligned}
$$

If $A$ is very general (outside a countable union of proper analytic subsets of $\left.\mathbb{L} \mathbb{G}\left(\wedge^{3} V\right)^{0} \cap \delta^{-1} \mathbb{L} \mathbb{G}\left(\wedge^{3} V^{\vee}\right)^{0}\right)$ then $W_{A}$ and $W_{A^{\perp}}$ are both indecomposable rational Hodge Structures - see Section (3) of [14]: since they contain the non-zero components $H^{4,0}\left(X_{A}\right)$ and $H^{4,0}\left(X_{A^{\perp}}\right)$ the above isomorphism defines an isomorphism of rational H.S.'s

$$
h_{A}: W_{A} \stackrel{\sim}{\longrightarrow} W_{A^{\perp}} .
$$

Let $Q_{A} \subset H^{2}\left(X_{A}\right)_{\text {prim }}$ and $Q_{A^{\perp}} \subset H^{2}\left(X_{A^{\perp}}\right)_{\text {prim }}$ be the cones of isotropic classes (with respect to the Beauville-Bogomolov bilinear form). Let

$$
\begin{array}{ccc}
H^{2}\left(X_{A}\right)_{\text {prim }} & \stackrel{\nu_{A}}{\longrightarrow} \operatorname{Sym}^{2} H^{2}\left(X_{A}\right)_{\text {prim }} \\
\alpha & \mapsto & \alpha^{2}
\end{array}
$$

be the (affine) Veronese map; we define similarly $\nu_{A^{\perp}}$. Then

$$
W_{A}=\operatorname{span} \nu_{A}\left(Q_{A}\right), \quad W_{A^{\perp}}=\operatorname{span} \nu_{A^{\perp}}\left(Q_{A^{\perp}}\right) .
$$

We claim that

$$
h_{A}\left(\nu_{A}\left(Q_{A}\right)\right)=\nu_{A^{\perp}}\left(Q_{A^{\perp}}\right) .
$$


In fact let $\mathcal{U} \subset \mathbb{L} \mathbb{G}\left(\wedge^{3} V\right)^{0} \cap \delta^{-1} \mathbb{L} \mathbb{G}\left(\wedge^{3} V^{\vee}\right)^{0}$ be an open ball containing $A$. The Gauss-Manin connection gives identifications

$$
H^{2}\left(X_{B}\right) \cong H^{2}\left(X_{A}\right), \quad H^{2}\left(X_{B^{\perp}}\right) \cong H^{2}\left(X_{A^{\perp}}\right)
$$

for every $B \in \mathcal{U}$. Gauss-Manin gives also identifications $W_{B} \cong W_{A}$ and $W_{B^{\perp}} \cong$ $W_{A^{\perp}} \cong$ sending $\nu_{B}\left(Q_{B}\right)$ to $\nu_{A}\left(Q_{A}\right)$ and $\nu_{B^{\perp}}\left(Q_{B^{\perp}}\right)$ to $\nu_{A^{\perp}}\left(Q_{A^{\perp}}\right)$ respectively. The isomorphism $h_{B}$ is flat for the Gauss-Manin connection hence it is identified with a (constant) map $h: W_{A} \rightarrow W_{A^{\perp}}$. For $B \in \mathcal{U}$ let $\sigma_{B}, \sigma_{B^{\perp}}$ be symplectic forms on $X_{B}$ and $X_{B^{\perp}}$ respectively. Since $h_{B}$ is an isomorphism of H.S.'s we have

$$
h\left[\sigma_{B}^{2}\right]=\left[\sigma_{B^{\perp}}^{2}\right] .
$$

Now $\sigma_{B} \in Q_{A}$ and $\sigma_{B^{\perp}} \in Q_{A^{\perp}}$ - here we make the identifications (4.1.16) - and as $B$ varies in $\mathcal{U}$ both $\left[\sigma_{B}\right]$ and $\left[\sigma_{B^{\perp}}\right]$ fill out non-empty open (in the classical topology) subsets of $\mathbb{P}\left(Q_{A}\right)$ and $\mathbb{P}\left(Q_{A^{\perp}}\right)$ respectively. Since $\mathbb{P}\left(Q_{A}\right)$ and $\mathbb{P}\left(Q_{A^{\perp}}\right)$ are non singular quadrics any non-empty open subset is Zariski-dense and hence (4.1.17) proves (4.1.15). It follows from (4.1.15) that there exists a linear map (4.1.3) welldefined up to \pm 1 such that for $\alpha \in Q_{A}$ we have $h_{A}\left(\alpha^{2}\right)=g_{A}(\alpha)^{2}$. By (4.1.15) we have

$$
g_{A}(\alpha) \in Q_{A^{\perp}} \text { if and only if } \alpha \in Q_{A} .
$$

The rationality of $g_{A}$ follows from the fact that $h_{A}$ is defined over $\mathbb{Q}$, and $\nu_{A}, \nu_{A^{\perp}}$ give bijective maps between $\mathbb{P}\left(Q_{A}\right)(\mathbb{Q}), \nu_{A}\left(\mathbb{P}\left(Q_{A}\right)\right)(\mathbb{Q})$ and $\mathbb{P}\left(Q_{A^{\perp}}\right)(\mathbb{Q})$, $\nu_{A^{\perp}}\left(\mathbb{P}\left(Q_{A^{\perp}}\right)\right)(\mathbb{Q})$ respectively. Finally let's prove that Equation (4.1.4) holds. First we show that

$$
(\alpha, \beta)_{X_{A}}=\left(g_{A}(\alpha), g_{A}(\beta)\right)_{X_{A^{\perp}}}, \quad \alpha, \beta \in Q_{A}
$$

Since (4.1.11) respects the intersection forms we have

$$
\int_{X_{A}} \alpha^{2} \wedge \beta^{2}=\int_{X_{A^{\perp}}} h_{A}\left(\alpha^{2}\right) \wedge h_{A}\left(\beta^{2}\right)=\int_{X_{A^{\perp}}} g_{A}(\alpha)^{2} \wedge g_{A}(\beta)^{2} .
$$


On the other hand $\alpha, \beta$ are isotropic and by (4.1.15) $g_{A}(\alpha), g_{A}(\beta)$ are isotropic as well; thus

$$
\begin{gathered}
\int_{X_{A}} \alpha^{2} \wedge \beta^{2}=2(\alpha, \beta)_{X_{A}}^{2} \\
\int_{X_{A^{\perp}}} g_{A}(\alpha)^{2} \wedge g_{A}(\beta)^{2}=2\left(g_{A}(\alpha), g_{A}(\beta)\right)_{X_{A^{\perp}}}^{2}
\end{gathered}
$$

(See Section (2) of [14].) Equations (4.1.20), (4.1.21) and (4.1.22) prove that either (4.1.19) holds or else

$$
(\alpha, \beta)_{X_{A}}=-\left(g_{A}(\alpha), g_{A}(\beta)\right)_{X_{A^{\perp}}}, \quad \alpha, \beta \in Q_{A} .
$$

Assume that (4.1.24) holds. Let $\sigma_{A} \in H^{2,0}\left(X_{A}\right)$ be the class of a symplectic forms on $X_{A}$; since $g_{A}$ is an isomorphism of Hodge structures $g_{A}\left(\sigma_{A}\right)$ is represenyted by a symplectic form on $X_{A^{\perp}}$. Then

$$
\left(\sigma_{A}, \overline{\sigma_{A}}\right)_{X_{A}}=-\left(g_{A}\left(\sigma_{A}\right), \overline{g_{A}\left(\sigma_{A}\right)}\right)_{X_{A^{\perp}}} .
$$

This is absurd because the Beaville-Bogomolov $(,)_{X}$ of an irreducible symplectic manifold $X$ has the property that $(\sigma, \bar{\sigma})_{X}>0$ for every symplectic form $\sigma$ on $X$. This finishes the proof of Equation (4.1.19). Now let $\gamma \in H^{2}\left(X_{A}\right)_{\text {prim }}$, then $\gamma=\alpha+\beta$ for certain $\alpha, \beta \in Q_{A}$; by Equations (4.1.18) and (4.1.19 we get

$$
(\gamma, \gamma)_{X_{A}}=2(\alpha, \beta)_{X_{A}}=2\left(g_{A}(\alpha), g_{A}(\beta)\right)_{X_{A^{\perp}}}=\left(g_{A}(\gamma), g_{A}(\gamma)\right)_{X_{A^{\perp}}}
$$

This proves (4.1.4).

Proposition (4.1) implies that locally there exists $g \in O(\Lambda \otimes \mathbb{Q})$ which relates the periods of $X_{A^{\perp}}$ to those of $X_{A}$ and furthermore $g$ normalizes the subgoup of monodromy operators in $O(\Lambda)$. We introduce some notation to formalize this observation. Let $\mathcal{U} \subset \mathbb{L} \mathbb{G}\left(\wedge^{3} V\right)^{\sharp}$ be a small open ball and let $\Psi, \Phi$ be markings of $\left.\mathbb{H}(V)\right|_{\mathcal{U}}$ and $\left.\mathbb{H}\left(V^{\vee}\right)\right|_{\delta(\mathcal{U})}$ respectively - see Definition (3.15). Let $\bar{A} \in \mathcal{U}$ be a reference point: the monodromy representation of $\pi_{1}\left(\mathbb{L} \mathbb{G}\left(\wedge^{3} V\right)^{\sharp}, \bar{A}\right)$ on $\mathbb{H}(V)$ determines via $\Psi_{\bar{A}}$ a monodromy representation $\pi_{1}\left(\mathbb{L} \mathbb{G}\left(\wedge^{3} V\right)^{\sharp}, \bar{A}\right) \rightarrow \operatorname{Stab}(u)$ where $\operatorname{Stab}(u)<O(\widetilde{\Lambda})$ is the subgroup fixing the element $u$ given by (1.0.8). An element of the image of the monodromy representation is a $\Psi_{\bar{A}}$-monodromy operator. Similarly we have a monodromy representation of $\pi_{1}\left(\mathbb{L} \mathbb{G}\left(\wedge^{3} V^{\vee}\right), \bar{A}\right)$ on $\mathbb{H}\left(V^{\vee}\right)$; this determines via $\Phi_{\bar{A}^{\perp}}$ a monodromy representation $\pi_{1}\left(\mathbb{L} \mathbb{G}\left(\wedge^{3} V^{\vee}\right)^{\sharp}, \bar{A}^{\perp}\right) \rightarrow \operatorname{Stab}(u)$. 
An element of the image of this second monodromy representation is a $\Phi_{\bar{A}^{\perp-}}$ monodromy operator. Here and in the rest of the paper we will adopt the following conventions. First we view both $O(\Lambda)$ and $O(\Lambda \otimes \mathbb{Q})$ as subgroups of $O(\Lambda \otimes \mathbb{C})$. Secondly if $\gamma \in O(\Lambda \otimes \mathbb{C})$ we denote by $\widetilde{\gamma} \in O(\widetilde{\Lambda} \otimes \mathbb{C})$ the isometry which fixes $u$ and equals $\gamma$ on $\Lambda$. As a rule letters decorated by a tilde denote elements of $O(\widetilde{\Lambda} \otimes \mathbb{C})$, letters with no tilde denote elements of $O(\Lambda \otimes \mathbb{C})$.

Corollary 4.2. Let $\mathcal{U} \subset \mathbb{L} \mathbb{G}\left(\wedge^{3} V\right)^{\sharp}$ be a small open ball and let $\Psi, \Phi$ be markings of $\left.\mathbb{H}(V)\right|_{\mathcal{U}}$ and $\left.\mathbb{H}\left(V^{\vee}\right)\right|_{\delta(\mathcal{U})}$ respectively. Let $\mathcal{P}_{\Psi}, \mathcal{P}_{\Phi}$ be the local period maps (3.3.12). There exists $g \in O(\Lambda \otimes \mathbb{Q})$ well-determined up to \pm 1 such that

$$
\mathcal{P}_{\Phi}\left(A^{\perp}\right)=g \circ \mathcal{P}_{\Psi}(A)
$$

for all $A \in \mathcal{U}$. Let $\widetilde{\gamma} \in \operatorname{Stab}(u)$ be a $\Psi_{\bar{A}}$-monodromy operator; then $\widetilde{g} \circ \widetilde{\gamma} \circ \widetilde{g}^{-1}$ is $a \Phi_{\bar{A}^{\perp}}$-monodromy operator, in particular $g \circ \gamma \circ g^{-1} \in O(\Lambda)$.

Proof. Equation (4.1.27) holds on $\mathcal{U} \cap \mathbb{L} \mathbb{G}\left(\wedge^{3} V\right)^{00}$ by Proposition (4.1) and flatness of $g_{A}$; by continuity Equation (4.1.27) holds on all of $\mathcal{U}$. The statement about monodromy operators holds by flatness of $g_{A}$.

4.2. Restriction to $\Delta_{*}^{0}(V)$ of the local period maps. Our next task is to analyze the restriction to $\Delta_{*}^{0}(V)$ of the local period maps $\mathcal{P}_{\Psi}$ and $\mathcal{P}_{\Phi}$.

Proposition 4.3. Let $\mathcal{U} \subset \mathbb{L} \mathbb{G}\left(\wedge^{3} V\right)^{\sharp}$ be a small open ball. There is a choice of markings $\Psi$ and $\Phi$ of $\left.\mathbb{H}(V)\right|_{\mathcal{U}}$ and $\left.\mathbb{H}\left(V^{\vee}\right)\right|_{\delta(\mathcal{U})}$ respectively such that

$$
\mathcal{P}_{\Psi}\left(\Delta_{*}^{0}(V) \cap \mathcal{U}\right)=\left(e_{1}+2 e_{2}\right)^{\perp} \cap \mathcal{P}_{\Psi}(\mathcal{U})
$$

where $e_{1}, e_{2} \in \Lambda$ are as in Section (1). Furthermore

$$
\mathcal{P}_{\Phi}\left(A^{\perp}\right)=r \circ \mathcal{P}_{\Psi}(A), \quad A \in \Delta_{*}^{0}(V)
$$

where $r$ is the involution defined by (2.2.12).

Proof. First we embed the lattice $\widetilde{\Lambda}$ in a unimodular lattice as follows. Let $\widehat{\Lambda}:=U^{4} \widehat{\oplus}\left(-E_{8}\right)^{2}$. Let $U_{1}<\widehat{\Lambda}$ be one of the hyperbolic lattices, let $z \in U_{1}$ be a vector of square 2 and $e_{2}$ be a generator of $z^{\perp} \cap U_{1}$. Then we have an isomorphism

$$
z^{\perp} \cong \widetilde{\Lambda}
$$

and we can choose it so that it matches the present $e_{2}$ with the vector $e_{2}$ appearing in (1.0.9): we fix such an isomorphism once for all. Let $u, e_{1} \in \widetilde{\Lambda}=z^{\perp}$ be as 
in Section (1); then $\left\{\left(u \pm e_{1}\right) / 2,\left(z \pm e_{2}\right) / 2\right\} \subset \widehat{\Lambda}$. Furthermore the sublattices $\left\langle\left(u+e_{1}\right) / 2,\left(u-e_{1}\right) / 2\right\rangle$ and $\left\langle\left(z+e_{2}\right) / 2,\left(z-e_{2}\right) / 2\right\rangle$ are orthogonal hyperbolic planes. Thus we have an orthogonal decomposition

$$
\widehat{\Lambda}=\left\langle\left(u+e_{1}\right) / 2,\left(u-e_{1}\right) / 2\right\rangle \widehat{\oplus}\left\langle\left(z+e_{2}\right) / 2,\left(z-e_{2}\right) / 2\right\rangle \widehat{\oplus} U^{2} \widehat{\oplus}\left(-E_{8}\right)^{2} .
$$

Now we pass to the geometry. Let $T_{*}$ be as in Subsection (3.2). Let $A \in \Delta_{*}^{0}(V) \cap$ $\mathcal{U}$; by definition there exists $t \in T_{*}$ such that

$$
M_{t} \cong X_{A} .
$$

Since $\mathcal{U}$ is a small open ball there exists a small open ball $\mathcal{V} \subset T_{*}$ such that if $t \in \mathcal{V}$ then (4.2.5) holds for some $A \in \Delta_{*}^{0}(V) \cap \mathcal{U}$ and conversely if $A \in \Delta_{*}^{0}(V) \cap \mathcal{U}$ then there exists $t \in \mathcal{V}$ such that (4.2.5) holds. Let $\kappa: \mathcal{S} \rightarrow T_{*}$ be the tautological family of $K 3$ surfaces parametrized by $T_{*}$. Let $t \in T_{*}$. Let $S_{t}=\kappa^{-1}(t), D_{t}$ etc. be as in Subsection (3.1) and $A_{t} \in \Delta_{*}^{0}(V) \cap \mathcal{U}$ such that $M_{t} \cong X_{A_{t}}$. Let $f_{t}: M_{t} \rightarrow Y_{A_{t}}$ be the double cover. Let $v_{t}, w_{t} \in H^{*}\left(S_{t}\right)$ be given by

$$
v_{t}:=2+c_{1}\left(D_{t}\right)+2 \eta_{t}, \quad w_{t}:=1-\eta_{t}
$$

where $\eta_{t} \in H^{4}\left(S_{t} ; \mathbb{Z}\right)$ is the orientation class. The Mukai map

$$
\theta_{v_{t}}: v_{t}^{\perp} \rightarrow H^{2}\left(M_{t}\right)
$$

is an isometry of Hodge structures - see Subsection (3.1). Furthermore one has

$$
c_{1}\left(f_{t}^{*} L_{A_{t}}\right)=\theta_{v_{t}}\left(\eta_{t}-1\right)=\theta_{v_{t}}\left(-w_{t}^{\vee}\right) .
$$

(See the line preceding (3.1.7) for the definition of $w_{t}^{\vee}$.) The local system $\left.R^{2} \kappa_{*} \mathbb{Z}\right|_{\mathcal{V}}$ is trivial because $\mathcal{V}$ is a small open ball. Thus there exist sections $\alpha, \beta \in$ $\Gamma\left(R^{2} \kappa_{*} \mathbb{Z} \mid \mathcal{V}\right)$ such that $c_{1}\left(D_{t}\right)=\alpha_{t}+5 \beta_{t}$ for all $t \in \mathcal{V}$. We define a trivialization

$$
\left.R \kappa_{*} \mathbb{Z}\right|_{\mathcal{V}}=\left.\left(R^{0} \kappa_{*} \mathbb{Z} \oplus R^{2} \kappa_{*} \mathbb{Z} \oplus R^{4} \kappa_{*} \mathbb{Z}\right)\right|_{\mathcal{V}} \stackrel{\Upsilon}{\longrightarrow} \mathcal{V} \times \widehat{\Lambda}
$$

as follows. For $t \in \mathcal{V}$ let

$$
\begin{array}{rrrr}
\Upsilon_{t}(1) & := & -u / 2+e_{1} / 2-z & +e_{2} \\
\Upsilon_{t}\left(\eta_{t}\right) & := & u / 2+e_{1} / 2-z & +e_{2} \\
\Upsilon_{t}\left(\alpha_{t}\right) & := & -2 e_{1} & +5 z / 2-3 e_{2} / 2 \\
\Upsilon_{t}\left(\beta_{t}\right) & := & z / 2 & -e_{2} / 2
\end{array}
$$

and let

$$
\left.\Upsilon_{t}\right|_{\left\{1, \eta_{t}, \alpha_{t}, \beta_{t}\right\}^{\perp}}:\left\{1, \eta_{t}, \alpha_{t}, \beta_{t}\right\}^{\perp} \stackrel{\sim}{\longrightarrow}\left\{\left(u \pm e_{1}\right) / 2,\left(z \pm e_{2}\right) / 2\right\}^{\perp}
$$


be an arbitrary isometry - notice that $\left\{1, \eta_{t}, \alpha_{t}, \beta_{t}\right\}^{\perp} \cong U^{2} \widehat{\oplus}\left(-E_{8}\right)^{2}$ is isometric to $\left\{\left(u \pm e_{1}\right) / 2,\left(z \pm e_{2}\right) / 2\right\}^{\perp}$ by (4.2.4). A straightforward computation shows that $\Upsilon_{t}$ is an isometry. The trivialization (4.2.9) is defined to have value $\Upsilon_{t}$ at $t \in \mathcal{V}$. Now notice that $\Upsilon_{t}\left(v_{t}\right)=z$ and hence we have an isometry $\Upsilon_{t} \circ \theta_{v_{t}}^{-1}: H^{2}\left(M_{t}\right) \stackrel{\sim}{\longrightarrow}$ $z^{\perp}=\widetilde{\Lambda}$. Since $\Upsilon_{t}\left(-w_{t}^{\vee}\right)=u$ Equation (4.2.8) gives that

$$
\Upsilon_{t} \circ \theta_{v_{t}}^{-1}\left(c_{1}\left(f_{t}^{*} L_{A_{t}}\right)\right)=u .
$$

Hence $\Upsilon_{t} \circ \theta_{v_{t}}^{-1}$ defines a marking of $\left(M_{t}, f_{t}^{*} L_{A_{t}}\right)$ for every $t \in \mathcal{V}$; since $\left.\mathbb{H}(V)\right|_{\mathcal{U}}$ is trivial there exists a marking $\Psi:\left.\mathbb{H}(V)\right|_{\mathcal{U}} \longrightarrow \mathcal{U} \times \widetilde{\Lambda}$ such that

$$
\Psi_{A_{t}}=\Upsilon_{t} \circ \theta_{v_{t}}^{-1}, \quad t \in \mathcal{V} .
$$

Equation (4.2.1) follows from Equation (3.1.11) and the equality

$$
\Psi_{A_{t}}\left(\theta_{v_{t}}\left(5+2 c_{1}\left(D_{t}\right)+5 \eta_{t}\right)\right)=\Upsilon_{t}\left(5+2 c_{1}\left(D_{t}\right)+5 \eta_{t}\right)=e_{1}+2 e_{2} .
$$

Next we define a marking $\Phi$ for $\left.\mathbb{H}\left(V^{\vee}\right)\right|_{\delta(\mathcal{U})}$. By (3.3.4) this will be equivalent to a marking of $S_{t}^{[2]}$, hence we first recall the description of $H^{2}\left(S_{t}^{[2]}\right)$. We notice that $w_{t}$ is the Mukai vector (see (3.1.1)) of any ideal sheaf $I_{Z}$ where $[Z] \in S_{t}^{[2]}$; Mukai's map

$$
\theta_{w_{t}}: w_{t}^{\perp} \rightarrow H^{2}\left(S_{t}^{[2]}\right)
$$

is an isomorphism of polarized Hodge structures and an isometry. Let $g_{t}: S_{t}^{[2]} \rightarrow$ $Y_{A_{t}^{\perp}}$ be the map defined by (3.2.7); by Subsection (5.3) of [12]

$$
c_{1}\left(g_{t}^{*} \mathcal{O}_{Y_{A_{t}^{\perp}}}(1)\right)=\theta_{w_{t}}\left(-2+c_{1}\left(D_{t}\right)-2 \eta_{t}\right)=\theta_{w_{t}}\left(-v_{t}^{\vee}\right) .
$$

We define a trivialization

$$
\left.R \kappa_{*} \mathbb{Z}\right|_{\mathcal{V}}=\left.\left(R^{0} \kappa_{*} \mathbb{Z} \oplus R^{2} \kappa_{*} \mathbb{Z} \oplus R^{4} \kappa_{*} \mathbb{Z}\right)\right|_{\mathcal{V}} \stackrel{\Theta}{\longrightarrow} \mathcal{V} \times \widehat{\Lambda}
$$

as follows. For $t \in \mathcal{V}$ let

$$
\begin{array}{rlrl}
\Theta_{t}(1) & :=u & -e_{1} & +z / 2-e_{2} / 2 \\
\Theta_{t}\left(\eta_{t}\right) & :=u & -e_{1} & -z / 2-e_{2} / 2 \\
\Theta_{t}\left(\alpha_{t}\right) & :=5 u / 2-3 e_{1} / 2 & -2 e_{2} \\
\Theta_{t}\left(\beta_{t}\right) & :=u / 2 & -e_{1} / 2 &
\end{array}
$$

and let the restriction of $\Theta_{t}$ to $\left\{1, \eta_{t}, \alpha_{t}, \beta_{t}\right\}^{\perp}$ be equal to the restriction of $\Upsilon_{t}$. A straightforward computation shows that $\Theta_{t}$ is an isometry. The trivialization (4.2.17) is defined to have value $\Theta_{t}$ at $t \in \mathcal{V}$. Now notice that $\Theta_{t}\left(w_{t}\right)=z$ 
and hence we have an isometry $\Theta_{t} \circ \theta_{w_{t}}^{-1}: H^{2}\left(S_{t}^{[2]}\right) \stackrel{\sim}{\longrightarrow} z^{\perp}=\widetilde{\Lambda}$. Since $\Theta_{t}\left(-v_{t}^{\vee}\right)=u$ Equation (4.2.16) gives that

$$
\Theta_{t} \circ \theta_{v_{t}}^{-1}\left(c_{1}\left(g_{t}^{*} \mathcal{O}_{Y_{A_{t}^{\perp}}}\right)\right)=u .
$$

Hence $\Theta_{t} \circ \theta_{w_{t}}^{-1}$ defines a marking of $\left(S_{t}^{[2]}, g_{t}^{*} \mathcal{O}_{Y_{A_{t}^{\perp}}}(1)\right)$ for every $t \in \mathcal{V}$; by $(3.3 .4)$ and triviality of $\left.\mathbb{H}\left(V^{\vee}\right)\right|_{\delta(\mathcal{U})}$ there exists a marking $\Phi:\left.\mathbb{H}\left(V^{\vee}\right)\right|_{\delta(\mathcal{U})} \longrightarrow \mathcal{U} \times \widetilde{\Lambda}$ such that

$$
\Phi_{A_{t}}=\Theta_{t} \circ \theta_{w_{t}}^{-1}, \quad t \in \mathcal{V} .
$$

Now let's prove (4.2.2). Equation (4.2.2) is equivalent to

$$
\mathcal{P}_{\Theta_{t} \circ \theta_{w_{t}}^{-1}}\left(S_{t}^{[2]}, g_{t}^{*} \mathcal{O}_{Y_{A_{t}^{\perp}}}(1)\right)=r \circ \mathcal{P}_{\Upsilon_{t} \circ \theta_{v_{t}}^{-1}}\left(M_{t}, f_{t}^{*} L_{A}\right), \quad t \in \mathcal{V}
$$

by Equation (3.3.13). Since $\theta_{v_{t}}$ and $\theta_{w_{t}}$ are isomorphism of Hodge structures the above equation may be rewitten as

$$
\Theta_{t}\left(H^{2,0}\left(S_{t}\right)\right)=r \circ \Upsilon_{t}\left(H^{2,0}\left(S_{t}\right)\right), \quad t \in \mathcal{V} .
$$

For $t \in \mathcal{V}$ let

$$
\begin{array}{ccc}
H^{*}\left(S_{t} ; \mathbb{Q}\right) & \stackrel{\Xi_{t}}{\longrightarrow} & H^{*}\left(S_{t} ; \mathbb{Q}\right) \\
\gamma & \mapsto-\gamma^{\vee}+\frac{1}{2}\left(\gamma^{\vee}, v_{t}+w_{t}\right)\left(v_{t}+w_{t}\right)
\end{array}
$$

i.e. the composition of the isometry $\gamma \mapsto \gamma^{\vee}$ and the reflection which is $(+1)$ on $\mathbb{Q}\left(v_{t}+w_{t}\right)$ and $(-1)$ on $\left(v_{t}+w_{t}\right)^{\perp}$; thus $\Xi_{t}$ is a rational (not integral !) Hodge isometry. A straightforward computation shows that $\Theta_{t}=r \circ \Upsilon_{t} \circ \Xi_{t}$. Thus (4.2.22) follows at once from $\Xi_{t}\left(H^{2,0}(S)\right)=H^{2,0}(S)$.

If $\gamma \in \Lambda \otimes \mathbb{Q}$ is non-isotropic we let

$$
\begin{aligned}
\Lambda \otimes \mathbb{Q} & \stackrel{r_{\gamma}}{\longrightarrow} \quad \Lambda \otimes \mathbb{Q} \\
x & \mapsto-x+\frac{2}{(\gamma, \gamma)}(x, \gamma) \gamma
\end{aligned}
$$

be the reflection with $(+1)$-eigenspace $\mathbb{Q} \gamma$ and $(-1)$-eigenspace $\gamma^{\perp}$. Let

$$
\zeta:=e_{1}+2 e_{2} .
$$

Corollary 4.4. Keep notation and assumptions of Proposition (4.3). Let $\Psi, \Phi$ be the markings of Proposition (4.3). Then:

(1) $\mathcal{P}_{\Phi}\left(A^{\perp}\right)=r \circ \mathcal{P}_{\Psi}(A)$ for all $A \in \mathcal{U}$ or

(2) $\mathcal{P}_{\Phi}\left(A^{\perp}\right)=r \circ r_{\zeta} \circ \mathcal{P}_{\Psi}(A)$ for all $A \in \mathcal{U}$. 
Proof. By Corollary (4.2) there exists $g \in O(\Lambda \otimes \mathbb{Q})$ such that $\mathcal{P}_{\Phi}\left(A^{\perp}\right)=g \circ \mathcal{P}_{\Psi}(A)$ for all $A \in \mathcal{U}$. By Proposition (4.3) $r^{-1} \circ g$ fixes the points of $\zeta^{\perp} \cap \mathcal{D}_{2}$ : it follows that $r^{-1} \circ g$ fixes $\zeta^{\perp} \subset \mathbb{P}(\Lambda \otimes \mathbb{C})$. Since $r \in O(\Lambda)$ we have $r^{-1} \circ g \in O(\Lambda \otimes \mathbb{Q})$ : thus we get that that

$$
\left.r^{-1} \circ g\right|_{\zeta^{\perp}}= \pm I d_{\zeta^{\perp}}
$$

Since $\zeta$ is non-isotropic (in fact $(\zeta, \zeta)=-10$ ) and $r^{-1} \circ g \in O(\Lambda \otimes \mathbb{Q}$ ) we get that $r^{-1} \circ g(\zeta)= \pm \zeta$. It follows that $r^{-1} \circ g= \pm I d$ or $r^{-1} \circ g= \pm r_{\zeta}$. Since $-I d$ acts trivially on $\mathcal{D}_{2} \subset \mathbb{P}(\Lambda)$ the corollary follows.

4.3. The proof. We will apply the monodromy statement of Corollary (4.2) in order to show that Item (2) of Corollary (4.4) can not hold. We will use the following result.

Claim 4.5. Let

$$
\xi=a_{1} e_{1}+a_{2} e_{2}+\nu \in \Lambda
$$

be a $(-2)$-vector i.e. $(\xi, \xi)=-2$ and assume that $r_{\zeta} \circ r_{\xi} \circ r_{\zeta} \in O(\Lambda)$. Then

$$
(\xi, \zeta) \equiv 0 \quad(\bmod 5)
$$

Proof. A tedious straightforward computation gives that

$$
\begin{aligned}
r_{\zeta} \circ r_{\xi} \circ r_{\zeta}\left(e_{1}\right)=\frac{1}{25} & \left(18 a_{1}^{2}-48 a_{1} a_{2}+32 a_{2}^{2}-25\right) e_{1}- \\
& \quad-\frac{1}{25}\left(24 a_{1}^{2}-14 a_{1} a_{2}-24 a_{2}^{2}\right) e_{2}-\frac{2}{5}\left(3 a_{1}-4 a_{2}\right) \nu
\end{aligned}
$$

Thus $18 a_{1}^{2}-48 a_{1} a_{2}+32 a_{2}^{2} \equiv 0(\bmod 25)$. Since

$$
18 a_{1}^{2}-48 a_{1} a_{2}+32 a_{2}^{2}=2\left(3 a_{1}-4 a_{2}\right)^{2}
$$

we get that $3 a_{1}-4 a_{2} \equiv 0(\bmod 5)$. This proves $(4.3 .2)$ because

$$
(\xi, \zeta)=-2 a_{1}-4 a_{2} \equiv 3 a_{1}-4 a_{2} \quad(\bmod 5) .
$$

Proposition 4.6. Keep notation and assumptions of Proposition (4.3). Let $\Psi$, $\Phi$ be the markings of Proposition (4.3) and $\bar{A} \in \mathcal{U} \cap \Delta_{*}^{0}(V)$. There exists a $(-2)$-vector $\xi \in \Lambda$ such that $-r_{\xi}$ is a $\Psi_{\bar{A}}$-monodromy operator and

$$
(\xi, \zeta) \not \equiv 0 \quad(\bmod 5)
$$


We grant the above proposition for the moment being and we proceed to prove Theorem (1.1). Let notation and assumptions be as in Proposition (4.3) and $\Psi$, $\Phi$ be the markings of Proposition (4.3). Then either (1) or (2) of Corollary (4.4) holds. Suppose that (2) holds; we will arrive at a contradiction. Let $\xi$ be as in Proposition (4.6): by Corollary (4.2) we have $-r \circ r_{\zeta} \circ r_{\xi} \circ r_{\zeta} \circ r \in O(\Lambda)$. Since $r \in O(\Lambda)$ we get that $r_{\zeta} \circ r_{\xi} \circ r_{\zeta} \in O(\Lambda)$ : this contradicts Claim (4.5) because of (4.3.6). Thus (1) of Corollary (4.4) holds. Let $\mathcal{U}^{00}:=\mathcal{U} \cap \mathbb{L} \mathbb{G}\left(\wedge^{3} V\right)^{00}$; then $\mathcal{U}^{00}$ is an open (in the euclidean topology) non-empty subset of $\mathbb{L} \mathbb{G}\left(\wedge^{3} V\right)^{00}$. Since (1) of Corollary (4.4) holds we have

$$
\left.\mathcal{P} \circ \delta\right|_{\mathcal{U}^{00}}=\left.\bar{r} \circ \mathcal{P}\right|_{\mathcal{U}^{00}} .
$$

Both $\mathcal{P} \circ \delta$ and $\bar{r} \circ \mathcal{P}$ are holomorphic maps with domain the connected manifold $\mathbb{L} \mathbb{G}\left(\wedge^{3} V\right)^{00}$; by analytic continuation we get that Theorem (1.1) holds.

Proof of Proposition (4.6) Let $F \subset \mathbb{P}^{3}$ be a smooth quartic, thus $F$ is a $K 3$ surface. We have a regular map

$$
\begin{aligned}
& F^{[2]} \stackrel{g}{\longrightarrow} \mathbb{G} r\left(1, \mathbb{P}^{3}\right) \subset \mathbb{P}^{5} \\
& {[Z] \mapsto \quad \operatorname{span}(Z)}
\end{aligned}
$$

and $c_{1}\left(g^{*} \mathcal{O}_{\mathbb{G} r\left(1, \mathbb{P}^{3}\right)}(1)\right)$ has square 2 for the Beauville-Bogomolov form. If $F$ does not contain lines the above map is finite and hence $g^{*} \mathcal{O}_{\mathbb{G} r\left(1, \mathbb{P}^{3}\right)}(1)$ is an ample line-bundle on $F^{[2]}$, if $F$ contains a line $R$ then $g^{*} \mathcal{O}_{\mathbb{G} r\left(1, \mathbb{P}^{3}\right)}(1)$ is big and nef but it restricts to the trivial line-bundle on the $\mathbb{P}^{2}$ given by $R^{(2)} \subset F^{[2]}$. Assume that $F$ does not contain lines; we proved in Section (6) of [12] that

$$
\left(F^{[2]}, g^{*} \mathcal{O}_{\mathbb{G} r\left(1, \mathbb{P}^{3}\right)}(1)\right) \text { is deformation equivalent to }\left(M_{t}, f_{t}^{*} L_{A_{t}}\right)
$$

where $t \in T$, i.e. there exists a polarized family of irreducible symplectic 4folds over a connected basis with one fiber isomorphic to $\left(F^{[2]}, g^{*} \mathcal{O}_{\mathbb{G} r\left(1, \mathbb{P}^{3}\right)}(1)\right)$ and another fiber isomorphic to $\left(M_{t}, f_{t}^{*} L_{A_{t}}\right)$. Using this result we will show that the monodromy operator on $F$ given by a suitable $(-2)$-class orthogonal to $c_{1}\left(\mathcal{O}_{F}(1)\right)$ gives rise to a $\Psi(\bar{A})$-monodromy operator for which (4.3.6) holds. Before proving this we must dive into the details of the proof of (4.3.9). Let $F_{0} \subset \mathbb{P}^{3}$ be a smooth quartic surface containing a line $R$ and with Picard number 2, i.e. $\operatorname{Pic}\left(F_{0}\right)=\mathbb{Z}\left[A_{0}\right] \oplus \mathbb{Z}[R]$ where $A_{0}$ is the (hyper)plane class. The divisor $\left(2 A_{0}-R\right)$ is very ample and $c_{1}\left(2 A_{0}-R\right)^{2}=10$; thus we have an embedding

$$
F_{0} \hookrightarrow\left|2 A_{0}-R\right|^{\vee} \cong \mathbb{P}^{6}
$$


as a linearly normal $K 3$ surface of degree 10 . Let

$$
w_{0}:=1+c_{1}\left(A_{0}\right)+\eta_{0}, \quad v_{0}:=2+c_{1}\left(2 A_{0}-R\right)+2 \eta_{0}
$$

where $\eta_{0} \in H^{4}\left(F_{0} ; \mathbb{Z}\right)$ is the orientation class. Let $M_{w_{0}}$ be the moduli space of torsion-free sheaves $\mathcal{G}$ on $F_{0}$ such that $v(\mathcal{G})=w_{0}$; every such sheaf is equal to $I_{Z} \otimes \mathcal{O}_{F_{0}}\left(A_{0}\right)$ for a unique $[Z] \in F_{0}^{[2]}$ and hence

$$
M_{w_{0}} \cong F_{0}^{[2]} \text {. }
$$

We let $L_{w_{0}}:=g_{0}^{*} \mathcal{O}_{\mathbb{G r} r\left(1, \mathbb{P}^{3}\right)}(1)$. Let $M_{v_{0}}$ be the moduli space of $\left(2 A_{0}-R\right)$ semistable sheaves $\mathcal{F}$ on $F_{0}$ such that $v(\mathcal{F})=v_{0}$. The moduli space $M_{v_{0}}$ is smooth because $\left(2 A_{0}-R\right)$ is $v_{0}$-generic (see Section (6) of [12]) and hence it is a deformation of $(K 3)^{[2]}$. Mukai's map gives isometries of Hodge structures

$$
\theta_{w_{0}}: w_{0}^{\perp} \stackrel{\sim}{\longrightarrow} H^{2}\left(M_{w_{0}}\right), \quad \theta_{v_{0}}: v_{0}^{\perp} \stackrel{\sim}{\longrightarrow} H^{2}\left(M_{v_{0}}\right) .
$$

One has (see p.1241 of [12])

$$
c_{1}\left(L_{w_{0}}\right)=\theta_{w_{0}}\left(\eta_{0}-1\right) .
$$

We let $L_{v_{0}}$ be the line-bundle on $M_{v_{0}}$ such that

$$
c_{1}\left(L_{v_{0}}\right)=\theta_{v_{0}}\left(\eta_{0}-1\right) .
$$

In Lemma (6.2) of [12] we considered the birational map $M_{w_{0}} \rightarrow M_{v_{0}}$ whose inverse

$$
\varphi: M_{v_{0}} \rightarrow M_{w_{0}}
$$

is the Mukai reflection defined by the $(-2)$-vector

$$
u_{0}:=\left(1+c_{1}\left(A_{0}-R\right)+\eta_{0}\right)
$$

(notice that $-r_{u_{0}}\left(v_{0}\right)=w_{0}$ ), see [16]. Since $M_{v_{0}}$ and $M_{w_{0}}$ are irreducible symplectic manifolds the birational map $\varphi$ induces an isomorphism of lattices $\varphi^{*}: H^{2}\left(M_{w_{0}}\right) \cong H^{2}\left(M_{v_{0}}\right)$. By Theorem (2.9) of [16] we have

$$
\varphi^{*} \theta_{w_{0}}(\alpha)=\theta_{v_{0}}\left(-r_{u_{0}}\left(v_{0}\right)\right),
$$

in particular by (4.3.14)-(4.3.15) we have

$$
\varphi^{*} L_{w_{0}} \cong L_{v_{0}} .
$$

The birational map $\varphi$ is the flop of

$$
\Pi_{w_{0}}:=R^{(2)} \subset F_{0}^{[2]}=M_{w_{0}} .
$$


It follows that $M_{v_{0}}$ contains $\Pi_{v_{0}}:=\Pi_{w_{0}}^{\vee}$ and from (4.3.19) we get that $L_{v_{0}}$ is big, nef and its restriction to $\Pi_{v_{0}}$ is trivial. Let $\mathcal{X} \rightarrow B_{v_{0}}$ be a representative for the deformation space of $\left(M_{v_{0}}, L_{v_{0}}\right)$, i.e. deformations of $M_{v_{0}}$ that "keep $c_{1}\left(L_{v_{0}}\right)$ of type $(1,1)$ ". Similarly let $\mathcal{X}^{\prime} \rightarrow B_{w_{0}}$ be a representative for the deformation space of $\left(M_{w_{0}}, L_{w_{0}}\right)$. We let $0 \in B_{v_{0}}$ and $0 \in B_{w_{0}}$ be the points corresponding to $\left(M_{v_{0}}, L_{v_{0}}\right)$ and $\left(M_{w_{0}}, L_{w_{0}}\right)$ respectively. Thus for each $q \in B_{v_{0}}$ the fiber $X_{q}$ of $\mathcal{X} \rightarrow B_{v_{0}}$ over $q$ has a line-bundle $L_{q}$ which is a deformation of $L_{v_{0}}$. Similarly for each $s \in B_{w_{0}}$ the fiber $X_{s}^{\prime}$ of $\mathcal{X}^{\prime} \rightarrow B_{w_{0}}$ over $s$ has a line-bundle $L_{s}^{\prime}$ which is a deformation of $L_{w_{0}}$. We may and will assume that $B_{v_{0}}, B_{w_{0}}$ are contractible and hence Gauss-Manin gives identifications

$$
H^{2}\left(X_{q}\right) \cong H^{2}\left(M_{v_{0}}\right), \quad H^{2}\left(X_{s}^{\prime}\right) \cong H^{2}\left(M_{w_{0}}\right), \quad q \in B_{v_{0}}, s \in B_{w_{0}}
$$

which match $c_{1}\left(L_{q}\right)$ to $c_{1}\left(L_{v_{0}}\right)$ and $c_{1}\left(L_{s}^{\prime}\right)$ to $c_{1}\left(L_{w_{0}}\right)$. Let $B\left(\Pi_{v_{0}}\right) \subset B_{v_{0}}$ be the locus parametrizing deformations $X_{q}$ which contain a deformation of $\Pi_{v_{0}}$, and similarly let $B\left(\Pi_{w_{0}}\right) \subset B_{w_{0}}$ be the locus parametrizing deformations of $X_{s}^{\prime}$ which contain a deformation of $\Pi_{w_{0}}$.

Claim 4.7. Keep notation as above. Then

$$
\begin{aligned}
\operatorname{cod}\left(B\left(\Pi_{w_{0}}\right), B_{w_{0}}\right) & =1, \\
\operatorname{cod}\left(B\left(\Pi_{v_{0}}\right), B_{v_{0}}\right) & =1 .
\end{aligned}
$$

Proof of the claim. Let's prove (4.3.22). Let $\operatorname{Def}\left(M_{w_{0}}\right)$ be a representative of the deformation space of $M_{w_{0}}$ with $0 \in \operatorname{Def}\left(M_{w_{0}}\right)$ corresponding to $M_{w_{0}}$. The Kodaira-Spencer map followed by the isomorphism $H^{1}\left(\Theta_{M_{w_{0}}}\right) \cong H^{1}\left(\Omega_{M_{w_{0}}}\right)$ defined by a symplectic form gives an isomorphism $\Theta_{0}\left(\operatorname{Def}\left(M_{w_{0}}\right)\right) \cong H^{1}\left(\Omega_{M_{w_{0}}}\right)$. The codimension- 1 subvariety $B_{w_{0}} \subset M_{w_{0}}$ has tangent space given by

$$
\Theta_{0}\left(B_{w_{0}}\right)=\left\{\alpha \in H^{1}\left(\Omega_{M_{w_{0}}}\right) \mid\left(\alpha, c_{1}\left(L_{w_{0}}\right)\right)_{M_{w_{0}}}=0\right\} .
$$

Let $\operatorname{Def}\left(M_{w_{0}}, \Pi_{w_{0}}\right) \subset \operatorname{Def}\left(M_{w_{0}}\right)$ be the subset of deformations of $M_{w_{0}}$ containing a deformation of $\Pi_{w_{0}}$. Of course we have

$$
B\left(\Pi_{w_{0}}\right)=B_{w_{0}} \cap \operatorname{Def}\left(M_{w_{0}}, \Pi_{w_{0}}\right) .
$$

By Voisin [15] we know that $\operatorname{Def}\left(M_{w_{0}}, \Pi_{w_{0}}\right)$ is smooth and

$$
\Theta_{0}\left(\operatorname{Def}\left(M_{w_{0}}, \Pi_{w_{0}}\right)\right)=\left\{\alpha \in H^{1}\left(\Omega_{M_{w_{0}}}\right)|\alpha|_{\Pi_{w_{0}}}=0\right\} .
$$


By (4.3.20) we have $\Pi_{w_{0}} \cong \mathbb{P}^{2}$ and hence $\operatorname{Def}\left(M_{w_{0}}, \Pi_{w_{0}}\right)$ is a codimension-1 subvariety of $\operatorname{Def}\left(M_{w_{0}}\right)$. We claim that

$$
\Theta_{0}\left(B_{w_{0}}\right) \neq \Theta_{0}\left(\operatorname{Def}\left(M_{w_{0}}, \Pi_{w_{0}}\right)\right) .
$$

In fact $c_{1}\left(L_{w_{0}}\right) \in \Theta_{0}\left(\operatorname{Def}\left(M_{w_{0}}, \Pi_{w_{0}}\right)\right)$ because $\Pi_{w_{0}}$ is contracted by the linear system $\left|L_{w_{0}}\right|$ while $c_{1}\left(L_{w_{0}}\right) \notin \Theta_{0}\left(B_{w_{0}}\right)$ because $\left(c_{1}\left(L_{w_{0}}\right), c_{1}\left(L_{w_{0}}\right)\right)_{M_{w_{0}}}=2 \neq 0$. Equation (4.3.22) follows from (4.3.25) and (4.3.27). The proof of (4.3.23) is similar (notice that $\Pi_{v_{0}}$ is contracted by the linear system $\left|L_{v_{0}}\right|$ because $\varphi$ is the flop of $\left.\Pi_{w_{0}}\right)$.

There is a natural isomorphism of germs

$$
\mu:\left(B_{w_{0}}, 0\right) \stackrel{\sim}{\longrightarrow}\left(B_{v_{0}}, 0\right)
$$

such that $\mu\left(B\left(\Pi_{w_{0}}\right)\right)=B\left(\Pi_{v_{0}}\right)$ and if $s \notin B\left(\Pi_{w_{0}}\right)$ then $\left(X_{\mu(s)}, L_{\mu(s)}\right) \cong\left(X_{s}^{\prime}, L_{s}^{\prime}\right)$. Let $s \in B_{w_{0}}$ be such that $\left(X_{s}^{\prime}, L_{s}^{\prime}\right) \cong\left(F^{[2]}, g^{*} \mathcal{O}_{\mathbb{G} r\left(1, \mathbb{P}^{3}\right)}(1)\right)$ where $F$ is a quartic containing no lines. Then $s \notin B\left(\Pi_{w_{0}}\right)$ and hence

$$
\left(X_{\mu(s)}, L_{\mu(s)}\right) \cong\left(X_{s}^{\prime}, L_{s}^{\prime}\right) \cong\left(F^{[2]}, g^{*} \mathcal{O}_{\mathbb{G} r\left(1, \mathbb{P}^{3}\right)}(1)\right)
$$

On the other hand there exists $q \in B_{v_{0}}$ such that $\left(X_{q}, c_{1}\left(L_{q}\right)\right) \cong\left(M_{t}, \theta_{t}\left(\eta_{t}-1\right)\right)$ for $t \in T_{*}$ because the parameter space for linearly normal $K 3$ surfaces of degree 10 (an open subset of the relevant Hilbert scheme) is irreducible - notice that $q \notin B\left(\Pi_{v_{0}}\right)$ because $\theta_{t}\left(\eta_{t}-1\right)$ is ample. Thus there exists $\bar{A} \in \Delta_{*}^{0}(V)$ such that

$$
\left(X_{\bar{A}}, L_{\bar{A}}\right) \cong\left(X_{q}, L_{q}\right)
$$

Let $\gamma \in H^{2}(F ; \mathbb{Z})$ be a $(-2)$-class orthogonal to $c_{1}\left(\mathcal{O}_{F}(1)\right)$. Then $\gamma$ determines a monodromy operator on $H^{2}\left(F^{[2]}\right)$; by (4.3.29) and (4.3.30) this monodromy operator can be identified with a monodromy operator on $X_{\bar{A}}$ because polarized deformation spaces of irreducible symplectic manifolds are smooth. Given the trivializations (4.3.21) the monodromy operator in question is equal to $-r_{\varphi^{*} \theta_{w_{0}}}\left(\gamma_{0}\right)$ - here $\gamma_{0} \in H^{2}\left(F_{0}\right)$ is the class corresponding to $\gamma$ (the second trivialization of (4.3.21) defines an identification $\left.H^{2}(F) \cong H^{2}\left(F_{0}\right)\right)$. The corresponding $\Psi_{\bar{A}^{-}}$ monodromy operator is equal to $-r_{\xi}$ where

$$
\xi:=\Psi_{\bar{A}}\left(\varphi^{*} \theta_{w_{0}}\left(\gamma_{0}\right)\right) \in \Lambda
$$


Claim 4.8. Keep notation as above. Let $\gamma_{0} \in c_{1}\left(A_{0}\right)^{\perp} \subset H^{2}\left(F_{0} ; \mathbb{Z}\right)$ be a (-2)class such that

$$
\int_{F_{0}} \gamma_{0} \wedge c_{1}(R) \not \equiv 0 \quad(\bmod 5)
$$

Then

$$
(\xi, \zeta) \not \equiv 0 \quad(\bmod 5)
$$

Proof of the claim. By (4.2.14) we must check that

$$
\left(\varphi^{*} \theta_{w_{0}}\left(\gamma_{0}\right), \theta_{v_{0}}\left(5+2 c_{1}\left(2 A_{0}-R\right)+5 \eta_{0}\right)\right) \not \equiv 0 \quad(\bmod 5) .
$$

By (4.3.18) this is equivalent to

$$
\left(-r_{u_{0}}\left(\gamma_{0}\right), 5+2 c_{1}\left(2 A_{0}-R\right)+5 \eta_{0}\right) \not \equiv 0 \quad(\bmod 5) .
$$

where $u_{0}$ is given by (4.3.17). A straightforward computation gives that

$$
\left(-r_{u_{0}}\left(\gamma_{0}\right), 5+2 c_{1}\left(2 A_{0}-R\right)+5 \eta_{0}\right)=-8 \int_{F_{0}} \gamma_{0} \wedge c_{1}(R)
$$

and hence we get (4.3.34).

Certainly there exists a class $\gamma_{0}$ satisfying the hypotheses of Claim (4.8): the vector $\xi \in \Lambda$ given by (4.3.31) satisfies the thesis of Proposition (4.6).

\section{REFERENCES}

1. A. Beauville, Variétes Kähleriennes dont la premiére classe de Chern est nulle, J. Differential geometry 18, 1983, pp. 755-782.

2. A. Beauville - R. Donagi, La variété des droites d'une hypersurface cubique de dimension 4, C. R. Acad. Sci. Paris Sér. I Math. 301, 1985, pp. 703-706.

3. G. Casnati - F. Catanese, Even sets of nodes are bundle symmetric, J. Diff. Geom. 47, 1997, pp. 237-256.

4. D. Eisenbud - S. Popescu - C. Walter, Lagrangian subbundles and codimension 3 subcanonical subschemes, Duke Math. J. 107, 2001, pp. 427-467.

5. V. A. Iskovskih, Fano 3-folds, I, Math. USSR Izvestija, Vol. 11, 1977, pp. 485-527.

6. A. Markman, Brill-Noether duality for moduli spaces of sheaves on K3 surfaces, J. Algebraic Geometry 10, 2002, pp. 623-694.

7. M. Maruyama, Moduli of stable sheaves II, J. Math. Kyoto Univ. 18, 1978, pp. 557614 . 
8. S. Mukai, Curves, K3 surfaces and Fano 3-folds of genus $\leq 10$, Algebraic Geometry and Commutative Algebra, Vol. I, Kinokuniya Tokyo, 1988, pp. 357-377.

9. S. Mukai, Moduli of vector bundles on K3 surfaces and symplectic manifolds, Sugaku Expos. 1, 1988, pp. 139-174.

10. V. V. Nikulin, Integral symmetric bilinear forms and some of their applications, Math. USSR Izvestija 14, 1980, pp. 103-167.

11. K. O'Grady, The weight-two Hodge structure of moduli spaces of sheaves on a K3 surface, J. of Algebraic Geom. 6, 1997, pp. 599-644.

12. K. O'Grady, Involutions and linear systems on holomorphic symplectic manifolds, GAFA 15, 2005, pp. 1223-1274.

13. K. O'Grady, Irreducible symplectic 4-folds and Eisenbud-Popescu-Walter sextics, Duke Math. J. 34, 2006, pp. 99-137.

14. K. O'Grady, Irreducible symplectic 4-folds numerically equivalent to $(K 3)^{[2]}$, math.AG/0504434, to appear on Commun. Contemp. Math.

15. C. Voisin, Sur la stabilité des sous-variétés lagrangiennes des variétés symplectiques holomorphes, Complex projective geometry (Trieste, 1989/Bergen, 1989), London Math. Soc. Lecture Note Ser. 179, Cambridge Univ. Press, Cambridge, 1992, pp. 294-303.

16. K. Yoshioka, Some examples of Mukai's reflections on K3 surfaces, J. reine angew. Math. 515, 1999, pp. 97-123.

\section{Kieran G. O'Grady}

Università di Roma "La Sapienza"

Dipartimento di Matematica "Guido Castelnuovo"

Piazzale Aldo Moro n. 5, 00185 Rome, Italy

E-mail: ogrady@mat.uniroma1.it 